(c) 2019. This manuscript version is made available under the CC-BY-NC-ND 4.0 license http:// creativecommons.org/licenses/by-nc-nd/4.0/

\title{
Integral inversion of GRAIL inter-satellite gravitational accelerations for regional recovery of the lunar gravitational field
}

\author{
M. Šprlák ${ }^{\mathrm{a}, *}$, S.-C. Han ${ }^{\mathrm{a}}$, W.E. Featherstone ${ }^{\mathrm{b}}$ \\ ${ }^{a}$ School of Engineering, Faculty of Engineering and Built Environment, University of Newcastle, University \\ Drive, Callaghan, NSW 2308, Australia, email: michal.sprlak@newcastle.edu.au, \\ shin-chan.han@newcastle.edu.au \\ ${ }^{b}$ School of Earth and Planetary Sciences, Curtin University of Technology, GPO Box U1987, Perth, WA 6845, \\ Australia, email: W.Featherstone@curtin.edu.au
}

\begin{abstract}
We present an integral-based approach for high-resolution regional recovery of the gravitational field in this article. We derive rigorous remove-compute-restore integral estimators relating the line-of-sight gravitational acceleration to an arbitrary order radial derivative of the gravitational potential. The integral estimators are composed of three terms, i.e., the truncated integration, the low-frequency line-of-sight gravitational acceleration, and the high-frequency truncation error (effect of the distant zones). We test the accuracy of the integral transformations and of the integral estimators in a closed-loop simulation over the Montes Jura region on the nearside of the Moon. In this way, we determine optimal sizes of integration radii and grid discretisation. In addition, we investigate the performance of the regional integral inversion with synthetic and realistic GRAIL observations. We demonstrate that the regional inversion results of the disturbing gravitational potential and its first order radial derivative in the Montes Jura mountain range are less contaminated by high-frequency noise than the global spherical harmonic models.
\end{abstract}

Keywords: Integral transformation, Green's function, Truncation error, Spherical cap, Satellite-to-satellite tracking, Doppler tracking, Inverse problem

\section{Introduction}

The gravitational field of the Moon has been mapped by several missions, such as the Soviet Luna (Akim, 1966), Lunar Orbiter (Muller and Sjogren, 1968), Apollo 15 and 16 sub-satellites (Sjogren et al., 1974), Clementine (Zuber et al., 1994), Lunar Prospector (Konopliv et al., 1998, 2001), SELenological and ENgineering Explorer (SELENE) (Namiki et al., 2009), and most recently by Gravity Recovery And Interior Laboratory (GRAIL) (Zuber et al., 2013). Analyses of tracking observations have led to numerous gravitational field models (GFMs); see (Konopliv et al., 2013, 2014; Lemoine et al., 2013, 2014; Klinger et al., 2014; Wirnsberger et al., 2019) and the references therein. The earlier missions only mapped GFMs on the nearside, but intersatellite tracking on SELENE and GRAIL has now permitted global GFMs. The most recent spherical harmonic expansions from GRAIL are available up to degree 1500, which makes the lunar gravitational field the most detailed among all planetary bodies.

Any global GFM representations prior to the SELENE and GRAIL missions required strong regularisation (e.g., Floberghagen et al., 1999; Floberghagen, 2002) that resulted in undesirable smoothing in the GFM over the nearside. As a consequence, regional approaches have been

\footnotetext{
*Tel.:+61 24921 8984, Fax: +61249216991

Email address: michal.sprlak@gmail.com (M. Šprlák)
} 
applied to attempt to improve lunar GFMs over the nearside (Sugano and Heki, 2004; Goossens et al., 2005a,b; Han, 2008; Han et al., 2009, 2011). Besides the most recent GFMs do not suffer from heterogeneous data coverage, improvements by local methods are still possible, as shown by Goossens et al. $(2012,2014)$ over the South Pole-Aitken basin on the farside. Regional modelling may also be attractive for practical reasons. For example, high-resolution GFMs in a desired area may be obtained by using moderately powered computers, whereas supercomputers are necessary for a high-resolution global spherical harmonic parametrisation.

In this article, we develop a new method for inverting the GRAIL inter-satellite tracking measurements and determine the gravitational potential and its radial derivative over a region on the Moon. Our approach has several noteworthy aspects: (1) the gravitational potential and its functionals are parametrised in space domain, as opposed to the spectral parametrisation used by the GRAIL project, (2) the Line-of-Sight (LoS) gravitational acceleration is formed by inter-satellite tracking and orbit data and directly used as observable, as opposed to inter-satellite range-rate used with numerical force integration, (3) the regional method allows regularisation that can be optimized and be region-specific, as opposed to the uniform global power law constraint (e.g., Kaula, 1966) used in the existing global solutions, and (4) the developed mathematical apparatus is general because it converts an arbitrary order derivative of the gravitational potential onto the LoS gravitational acceleration. We present new gravitational field solutions with the equivalent resolution (corresponding to degree 1400) to the latest development by the NASA's GRAIL project (e.g., Zuber et al., 2013). Our solutions are the first high-resolution GRAIL gravitational field solutions processed independently by the users outside from the NASA GRAIL team.

We acknowledge numerous existing regional modelling methods (e.g., Rummel, 1980; Barriot and Balmino, 1992; Thalhammer, 1994; Freeden et al., 2002; Garcia, 2002; Novák, 2007; Schmidt et al., 2007; Bentel et al., 2013; Gruber et al., 2014; Eshagh and Šprlák, 2016). These are relevant to regional analysis approaches for satellite tracking observations of Earth-orbiting satellites, such as the CHAllenging Mini-satellite Payload (CHAMP) (Reigber et al., 2002) and Gravity Recovery And Climate Experiment (GRACE) (Tapley et al., 2004), and of satellites that orbited other planets. However, lunar gravitational field determination from GRAIL is more challenging, because we aim for high-resolution regional fields (corresponding to degree 1400) from spacecraft orbiting at altitudes from $10 \mathrm{~km}$ to $90 \mathrm{~km}$ above the Moon's surface.

\section{Preliminaries}

\subsection{Nomenclature and reference frames}

The reference frame is the planetocentric Cartesian coordinate system. It is defined by the origin $O$ at the centre of mass of the Moon and the orthonormal base vectors $\mathbf{e}_{x}$ (pointing to the intersection of the prime meridian with the equator), $\mathbf{e}_{y}$ (pointing such that the frame is right-handed), and $\mathbf{e}_{z}$ (pointing in the direction of the spin axis), see Fig. 1. The position vector $\mathbf{x}$ of an arbitrary point is:

$\mathbf{x}=\mathbf{x}(r, \Omega)=x(r, \Omega) \mathbf{e}_{x}+y(r, \Omega) \mathbf{e}_{y}+z(r, \Omega) \mathbf{e}_{z}$,

where $(x, y, z)$ are the planetocentric Cartesian coordinates. As a spherical geometry is exploited extensively in this article, we prefer to use planetocentric spherical coordinates as variables. These are given by the planetocentric radius $r$ and the angular planetocentric spherical direction $\Omega=(\varphi, \lambda)$, which substitutes the spherical latitude $\varphi$ and the longitude $\lambda$.

We also specify the local North-oriented reference frame (LNORF). This is given by a moving origin and the orthonormal right-handed basis composed of vectors $\mathbf{e}_{r}$ (pointing radially outward), $\mathbf{e}_{\varphi}$ (pointing to the North), and $\mathbf{e}_{\lambda}$ (pointing to the West); see Fig. 1. The base vectors 


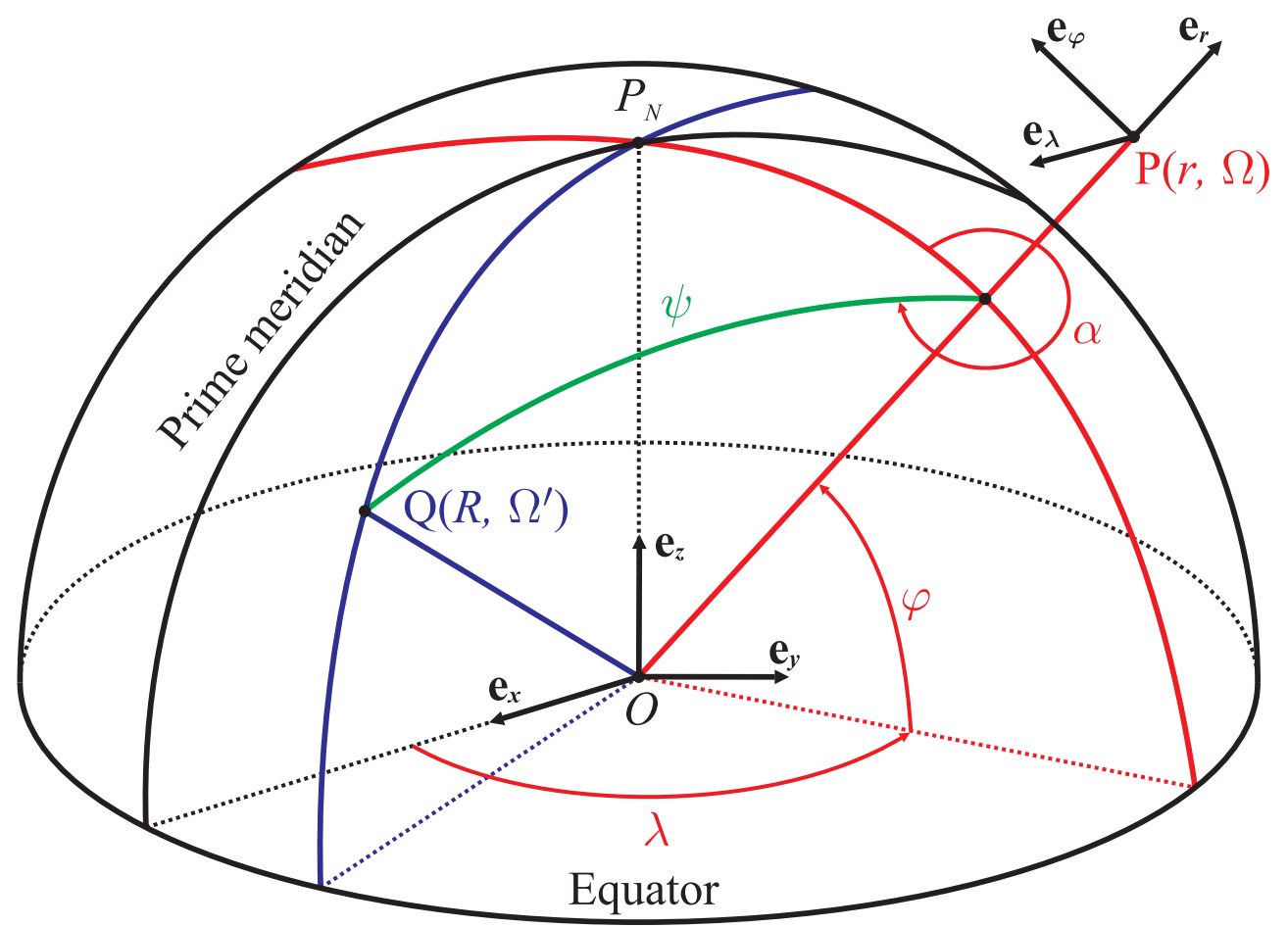

Figure 1: Illustration of reference frames and coordinates. $O$ is the origin of the planetocentric Cartesian reference frame. The point $\mathrm{P}$ is an evaluation point and the point $\mathrm{Q}$ represents an integration point. $P_{N}$ stands for the lunar North pole.

$\left(\mathbf{e}_{r}, \mathbf{e}_{\varphi}, \mathbf{e}_{\lambda}\right)$ change with the angular planetocentric spherical direction $\Omega$ and are related to the planetocentric Cartesian base vectors $\left(\mathbf{e}_{x}, \mathbf{e}_{y}, \mathbf{e}_{z}\right)$ as (Simmonds, 1994, p. 57):

$\mathbf{e}_{r}(\Omega)=\cos \varphi \cos \lambda \mathbf{e}_{x}+\cos \varphi \sin \lambda \mathbf{e}_{y}+\sin \varphi \mathbf{e}_{z}$,

$\mathbf{e}_{\varphi}(\Omega)=-\sin \varphi \cos \lambda \mathbf{e}_{x}-\sin \varphi \sin \lambda \mathbf{e}_{y}+\cos \varphi \mathbf{e}_{z}$

$\mathbf{e}_{\lambda}(\Omega)=\sin \lambda \mathbf{e}_{x}-\cos \lambda \mathbf{e}_{y}$

We distinguish two basic types of points; see Fig. 1: 1) We evaluate gravitational field quantities at a computation point, designated by the spherical triplet $(r, \Omega) ; 2)$ An integration point defines the position of a surface integration element defined by the planetocentric spherical coordinates $\left(R, \Omega^{\prime}\right)$. The symbol $R$ stands for the radius of the Brillouin sphere, which fully encloses all gravitating masses of an attracting body (e.g., Sansò and Sideris, 2013, Sect. 3.2).

Two substitutions are used throughout this article:

$t=t(r, R)=\frac{R}{r}, \quad r \geq R$

$u=u\left(\Omega, \Omega^{\prime}\right)=\cos \psi=\sin \varphi \sin \varphi^{\prime}+\cos \varphi \cos \varphi^{\prime} \cos \left(\lambda^{\prime}-\lambda\right)$.

The symbol $\psi$ represents the spherical distance between the computational point and the integration element. The two substitutions of Eqs. (5) and (6) are often complemented by the direct/forward azimuth $\alpha$, which is measured clockwise from North; see Fig. 1. 


\subsection{Radial boundary-value problem and its analytical solution}

The disturbing gravitational potential $T=W-U$, where $W$ is the actual gravity potential and $U$ is the normal gravity potential, is a fundamental scalar quantity of the gravitational field. The radial boundary-value problem (BVP) aims at determining $T$ on the surface of and outside the Brillouin sphere; $T$ thus fulfils Laplace's equation. The boundary condition $\mathcal{T}^{k}$, i.e., the $k$-th order radial derivative of $T$, is continuous on the Brillouin sphere. In addition, the regularity condition is valid at infinity. Mathematically, we can formulate the radial BVP as:

$\nabla^{2} T(r, \Omega)=0, \forall r>R$,

$\mathcal{T}^{k}(r, \Omega)=\frac{\partial^{k} T(r, \Omega)}{\partial r^{k}}, \forall r=R$

$\lim _{r \rightarrow \infty} T(r, \Omega)=0$

The values of $k$ in Eq. (8) are restricted to non-negative integers, i.e., $k \in \mathbb{Z}^{*}$. These specify the boundary conditions that can be expanded in terms of spherical harmonics as:

$\mathcal{T}^{k}(R, \Omega)=\sum_{n=0}^{\infty} \mathcal{T}_{n}^{k}(R, \Omega)$

with the corresponding $n$-th degree spherical harmonic:

$\mathcal{T}_{n}^{k}(R, \Omega)=(-1)^{k} \frac{G M}{a^{k+1}}\left(\frac{a}{R}\right)^{n+k+1} \frac{(n+k) !}{n !} \sum_{m=-n}^{+n} \Delta \bar{C}_{n, m} \bar{Y}_{n, m}(\Omega)$,

where $G$ stands for the universal gravitational constant, $M$ is the mass of the gravitating body, $a$ represents the major semi-axis of the reference ellipsoid, $\Delta \bar{C}_{n, m}$ is the $4 \pi$ fully normalised spherical harmonic coefficient of degree $n$ and order $m$ (reduced by the corresponding coefficient of the normal gravitational field), and $\bar{Y}_{n, m}$ is the $4 \pi$ normalised spherical harmonic.

An analytical solution of the radial BVP of Eqs. (7)-(9) can be found in the form of the integral transformation:

$T(r, \Omega)=(-1)^{k} \frac{R^{k}}{4 \pi} \int_{\Omega^{\prime}} \mathcal{T}^{k}\left(R, \Omega^{\prime}\right) \mathcal{H}_{k}(t, u) \mathrm{d} \Omega^{\prime}$.

The boundary condition $\mathcal{T}^{k}$ is weighted by the integral kernel (Green's function) $\mathcal{H}_{k}$, which is isotropic, i.e., it depends only on the spherical distance $\psi$, and given the value of $t$. The integral kernel can be defined compactly by the spectral relation:

$\mathcal{H}_{k}(t, u)=\sum_{n=0}^{\infty} t^{n+1} \frac{(2 n+1) n !}{(n+k) !} P_{n, 0}(u)$

where $P_{n, 0}$ is the un-normalised associated Legendre function of the first kind (also known as the Legendre polynomial of $n$-th degree).

Solutions to the radial BVP of Eqs. (7)-(9) represent the Abel-Poisson integral for $k=0$ (Kellogg, 1929, pp. 240-242) and the extended Hotine integral for $k=1$ (Hotine, 1969, Chap. 29). Martinec (2003) provided the analytical solution for the vertical-vertical gradiometric BVP that corresponds to $k=2$. In addition, Šprlák and Novák (2016) found the analytical solution for the vertical-vertical-vertical gravitational curvature BVP being consistent with $k=3$. Spatial forms of the isotropic kernels for these radial BVPs have also been provided, see Appendix A. 


\subsection{GRAIL mission observables}

GRAIL (Zuber et al., 2013) was launched on 10 September 2011. It consisted of two and almost identical spacecraft called Ebb (GRAIL-A) and Flow (GRAIL-B). They arrived at and started orbiting the Moon on 31 December 2011 and 1 January 2012, respectively.

Mapping the lunar gravitational field by GRAIL was performed in two phases. The Primary Mission started on 1 March 2012 and ended on 29 May 2012. The satellites orbited at an average altitude of $\sim 55 \mathrm{~km}$ above the lunar surface and their separation varied from $80 \mathrm{~km}$ to $220 \mathrm{~km}$. The Extended Mission began on 30 August 2012 and terminated on 14 December 2012. Tracking data were collected at an average altitude of $\sim 20 \mathrm{~km}$ and as low as $\sim 11 \mathrm{~km}$, with an inter-satellite distance of $30 \mathrm{~km}$ and $75 \mathrm{~km}$, towards the end of the mission.

The GRAIL observables were collected by the Deep Space Network (DSN) and the Lunar Gravity Ranging System (LGRS). The DSN provided the one-way X band and the two-way S band Doppler tracking data. These were processed and transformed into the positions of Ebb and Flow in the planetocentric Cartesian reference system by NASA (cf. Eq. (1)). In addition, the velocity and acceleration vectors were obtained by differentiating the planetocentric position with respect to time, i.e.:

$\dot{\mathbf{x}}=\dot{\mathbf{x}}(r, \Omega)=\dot{x}(r, \Omega) \mathbf{e}_{x}+\dot{y}(r, \Omega) \mathbf{e}_{y}+\dot{z}(r, \Omega) \mathbf{e}_{z}$,

$\ddot{\mathbf{x}}=\ddot{\mathbf{x}}(r, \Omega)=\ddot{x}(r, \Omega) \mathbf{e}_{x}+\ddot{y}(r, \Omega) \mathbf{e}_{y}+\ddot{z}(r, \Omega) \mathbf{e}_{z}$

The LGRS exploited the Ka-band microwave ranging between the two GRAIL satellites. Its measurements resulted in a time series of the inter-satellite range $\rho$, range-rate $\dot{\rho}$, and rangeacceleration $\ddot{\rho}$ (Hajela, 1979; Rummel, 1980):

$\rho=\rho\left(r_{1}, \Omega_{1}, r_{2}, \Omega_{2}\right)=\left|\mathbf{x}\left(r_{2}, \Omega_{2}\right)-\mathbf{x}\left(r_{1}, \Omega_{1}\right)\right|$,

$\dot{\rho}=\dot{\rho}\left(r_{1}, \Omega_{1}, r_{2}, \Omega_{2}\right)=\left[\dot{\mathbf{x}}\left(r_{2}, \Omega_{2}\right)-\dot{\mathbf{x}}\left(r_{1}, \Omega_{1}\right)\right] \cdot \mathbf{b}$,

$\ddot{\rho}=\ddot{\rho}\left(r_{1}, \Omega_{1}, r_{2}, \Omega_{2}\right)=\left[\ddot{\mathbf{x}}\left(r_{2}, \Omega_{2}\right)-\ddot{\mathbf{x}}\left(r_{1}, \Omega_{1}\right)\right] \cdot \mathbf{b}+\frac{\left|\dot{\mathbf{x}}\left(r_{2}, \Omega_{2}\right)-\dot{\mathbf{x}}\left(r_{1}, \Omega_{1}\right)\right|^{2}-\dot{\rho}^{2}}{\rho}$.

Here, the subscripts 1 and 2 distinguish the Ebb and Flow spacecraft, respectively. The symbol b stands for the unit inter-satellite LoS vector, defined as:

$\mathbf{b}=\frac{\mathbf{x}\left(r_{2}, \Omega_{2}\right)-\mathbf{x}\left(r_{1}, \Omega_{1}\right)}{\rho}=b_{x} \mathbf{e}_{x}+b_{y} \mathbf{e}_{y}+b_{z} \mathbf{e}_{z}$,

with $b_{x}=b_{x}\left(r_{1}, \Omega_{1}, r_{2}, \Omega_{2}\right)=\left(x_{2}-x_{1}\right) / \rho, b_{y}=b_{y}\left(r_{1}, \Omega_{1}, r_{2}, \Omega_{2}\right)=\left(y_{2}-y_{1}\right) / \rho$, and $b_{z}=$ $b_{z}\left(r_{1}, \Omega_{1}, r_{2}, \Omega_{2}\right)=\left(z_{2}-z_{1}\right) / \rho$ being the components of $\mathbf{b}$ in the planetocentric Cartesian reference frame.

The DSN and LGRS measurements have to be related to the disturbing gravitational potential $T$ to perform any modelling. The required mathematical connection provides the LoS disturbing gravitational acceleration, i.e., the difference of the disturbing gravitational gradients projected onto the LoS (e.g., Hajela, 1979; Rummel, 1980; Garcia, 2002; Keller and Sharifi, 2005):

$$
\begin{aligned}
& {\left[\nabla T\left(r_{2}, \Omega_{2}\right)-\nabla T\left(r_{1}, \Omega_{1}\right)\right] \cdot \mathbf{b}=\ddot{\rho}-\frac{\left|\dot{\mathbf{x}}\left(r_{2}, \Omega_{2}\right)-\dot{\mathbf{x}}\left(r_{1}, \Omega_{1}\right)\right|^{2}-\dot{\rho}^{2}}{\rho}} \\
& -\left[\nabla U\left(r_{2}, \Omega_{2}\right)-\nabla U\left(r_{1}, \Omega_{1}\right)\right] \cdot \mathbf{b} .
\end{aligned}
$$


The last term on the right-hand side of Eq. (20) is the effect of the normal (reference) gravitational field.

Han (2013) derived an identical formulation, but replaced the second term on the righthand side of our Eq. (20) with the inter-satellite velocity vectors. He also provided an error analysis of computing LoS gravitational acceleration considering range acceleration measurement noise, orbital state vector error, misalignment error, effects of non-conservative forces and timevariable gravitational acceleration. He found that the range acceleration measurement noise is the dominant error source in LoS gravitational acceleration at high frequencies $(>0.01 \mathrm{~Hz}$ that corresponds to the spherical harmonic degree greater than $\sim 100$ ). In the subsequent sections, we present a method of inverting LoS gravitational acceleration computed after Han (2013) and results from actual GRAIL range acceleration and orbital state vector data.

\section{Mathematical model for inverting LoS gravitational acceleration}

\subsection{Integral transformations}

We show the mathematical link between the LoS disturbing gravitational acceleration and the radial derivatives of the disturbing gravitational potential. For this purpose, we rewrite the differential operator on the left-hand side of Eq. (20) as follows (e.g., Šprlák and Novák, 2014):

$\delta[\nabla \bullet] \cdot \mathbf{b}=\delta\left[b_{r} \frac{\partial}{\partial r}+b_{\varphi} \frac{1}{r} \frac{\partial}{\partial \varphi}-b_{\lambda} \frac{1}{r \cos \varphi} \frac{\partial}{\partial \lambda}\right]$

$=\frac{1}{R} \delta\left[-b_{r} t^{2} \frac{\partial}{\partial t}+\left(b_{\varphi} \cos \alpha-b_{\lambda} \sin \alpha\right) t \sqrt{1-u^{2}} \frac{\partial}{\partial u}\right]$,

where $\delta$ represents the difference of the same function at two different locations $\left(r_{1}, \Omega_{1}\right)$ and $\left(r_{2}, \Omega_{2}\right)$. The function, to which the gradient (nabla) operator is applied, is substituted by the bullet on the left-hand side of Eq. (21). We omit the bullet symbol on the right-hand side, as the action of the partial derivatives on the function is implicit.

Equation (21) specifies the LoS gravitational differential operator in the LNORF. The unit inter-satellite LoS vector and the gradient operator in this reference frame are (e.g., Casotto and Fantino, 2009):

$\mathbf{b}=b_{r} \mathbf{e}_{r}+b_{\varphi} \mathbf{e}_{\varphi}+b_{\lambda} \mathbf{e}_{\lambda}, \quad \nabla=\frac{\partial}{\partial r} \mathbf{e}_{r}+\frac{1}{r} \frac{\partial}{\partial \varphi} \mathbf{e}_{\varphi}-\frac{1}{r \cos \varphi} \mathbf{e}_{\lambda}$.

The components of $\mathbf{b}$ in the LNORF, i.e., the scalars $b_{r}=b_{r}\left(r_{1}, \Omega_{1}, r_{2}, \Omega_{2}\right), b_{\varphi}=b_{\varphi}\left(r_{1}, \Omega_{1}, r_{2}, \Omega_{2}\right)$, and $b_{\lambda}=b_{\lambda}\left(r_{1}, \Omega_{1}, r_{2}, \Omega_{2}\right)$, differ from their counterparts $\left(b_{x}, b_{y}, b_{z}\right)$ in the planetocentric Cartesian coordinate system. The transformation between the components $\left(b_{x}, b_{y}, b_{z}\right)$ and $\left(b_{r}, b_{\varphi}, b_{\lambda}\right)$ can be found by substituting $b_{i} \rightarrow \mathbf{e}_{i}, i \in\{x, y, z\}$, and $b_{\mu} \rightarrow \mathbf{e}_{\mu}, \mu \in\{r, \varphi, \lambda\}$, in Eqs. (2)-(4). We also note that the orientation of the LNORFs at Ebb and Flow is not identical. Therefore, the components $\left(b_{r}, b_{\varphi}, b_{\lambda}\right)$ have to be computed separately for each of the GRAIL satellites.

The expression after the first equality in Eq. (21) defines the differential operator of the LoS gravitational acceleration in terms of the planetocentric spherical coordinates $(r, \Omega)$. This form is of utility when working with spherical harmonic expansions. For the GFM by integral transformations, representation in terms of the variables $(t, u, \alpha)$, given by the last expression of Eq. (21), is more suitable. Applying this form of the differential operator to the analytical solution of the radial BVP of Eq. (12), we obtain:

$\delta[\nabla T(r, \Omega)] \cdot \mathbf{b}=(-1)^{k} \frac{R^{k}}{4 \pi} \int_{\Omega^{\prime}} \mathcal{T}^{k}\left(R, \Omega^{\prime}\right) \delta\left[\nabla \mathcal{H}_{k}(t, u)\right] \cdot \mathbf{b} \mathrm{d} \Omega^{\prime}$ 
$=(-1)^{k} \frac{R^{k-1}}{4 \pi} \int_{\Omega^{\prime}} \mathcal{T}^{k}\left(R, \Omega^{\prime}\right) \delta\left[b_{r} \mathcal{H}_{k}^{t}(t, u)+\left(b_{\varphi} \cos \alpha-b_{\lambda} \sin \alpha\right) \mathcal{H}_{k}^{u}(t, u)\right] \mathrm{d} \Omega^{\prime}$.

The last equation represents the mathematical relationship between the LoS disturbing gravitational acceleration and the radial derivatives of the disturbing gravitational potential.

The resulting sub-integral kernel $\delta\left[\nabla \mathcal{H}_{k}\right] \cdot \mathbf{b}$ is a complicated mathematical function of three points, i.e., the two GRAIL spacecraft $\left(r_{1}, \Omega_{1}\right),\left(r_{2}, \Omega_{2}\right)$, and the integration point $\left(R, \Omega^{\prime}\right)$. We can decompose the sub-integral kernel into three independent parts. The kernels $\mathcal{H}_{k}^{t}$ and $\mathcal{H}_{k}^{u}$ represent the first, isotropic, part and read:

$\mathcal{H}_{k}^{t}(t, u)=-t^{2} \frac{\partial}{\partial t} \mathcal{H}_{k}(t, u)=-\sum_{n=0}^{\infty} t^{n+2} \frac{(2 n+1)(n+1) n !}{(n+k) !} P_{n, 0}(u)$

$\mathcal{H}_{k}^{u}(t, u)=t \sqrt{1-u^{2}} \frac{\partial}{\partial u} \mathcal{H}_{k}(t, u)=\sum_{n=1}^{\infty} t^{n+2} \frac{(2 n+1) n !}{(n+k) !} P_{n, 1}(u)$

The spatial (closed) forms of the isotropic kernel functions and their limiting values for $k=$ 0,1,2,3 are listed in Appendix A.

The second, non-isotropic (i.e., azimuth-dependent) part of the sub-integral kernel $\delta\left[\nabla \mathcal{H}_{k}\right] \cdot \mathbf{b}$ originates from the trigonometric functions of $\alpha$, which are (e.g., Chauvenet, 1875, pp. 151-154):

$\cos \alpha=\frac{1}{\sqrt{1-u^{2}}}\left[\sin \varphi^{\prime} \cos \varphi-\cos \varphi^{\prime} \sin \varphi \cos \left(\lambda^{\prime}-\lambda\right)\right]$,

$\sin \alpha=\frac{1}{\sqrt{1-u^{2}}} \cos \varphi^{\prime} \sin \left(\lambda^{\prime}-\lambda\right)$.

The third, heterogeneous (i.e., position-dependent) part is attributed to the unit inter-satellite LoS vector components $\left(b_{r}, b_{\varphi}, b_{\lambda}\right)$.

The sub-integral kernel $\delta\left[\nabla \mathcal{H}_{0}\right] \cdot \mathbf{b}$ is depicted in Fig. 2 as an example of its complicated spatial behaviour. We selected one representative formation of the two GRAIL satellites from the Extended Mission that defines the locations of the computational points. Here, the planetocentric spherical coordinates of Ebb are $r_{1} \approx 1753544.3 \mathrm{~m}, \varphi_{1} \approx 44.31^{\circ}, \lambda_{1} \approx 320.27^{\circ}$, while those of Flow are $r_{2} \approx 1753688.3 \mathrm{~m}, \varphi_{2} \approx 46.08^{\circ}, \lambda_{2} \approx 320.25^{\circ}$. The sub-integral kernel function was computed in the area $\varphi^{\prime} \in\left[42^{\circ}, 48^{\circ}\right]$ and $\lambda^{\prime} \in\left[-53^{\circ},-27^{\circ}\right]$ with the equiangular steps $\Delta \varphi^{\prime}=\Delta \lambda^{\prime}=0.01^{\circ}$. This fine spatial resolution was chosen simply for illustrative purposes in Fig. 2. We employed the isotropic kernels $\mathcal{H}_{0}^{t}$ and $\mathcal{H}_{0}^{u}$ by the spatial forms of Eqs. (A.5) and (A.11) in the calculations.

Figure 2 reveals the azimuthal dependence of the kernel $\delta\left[\nabla \mathcal{H}_{0}\right] \cdot \mathbf{b}$. The most pronounced values occur in the along-track direction, which almost coincides with the direction of the spherical latitude. The kernel values are zero at the locations of Ebb and Flow. The local extrema can be found in the distance of few tenths of arc-degrees from the computational points. The kernel values then decrease or increase to zero when increasing the distance from Ebb and Flow. We can also see that the magnitudes of the sub-integral kernel are reduced in the cross-track (longitude) direction.

Similar spatial behaviour can be observed for the GRAIL satellites at higher altitudes, e.g., such as for the Primary Mission, but the corresponding kernel is much smoother. The subintegral kernel functions for the higher-order radial derivatives of the disturbing gravitational potential are also characterised by lower spatial gradients. In particular, their zero crossings occur further from the computation points. These cases are not plotted here. 


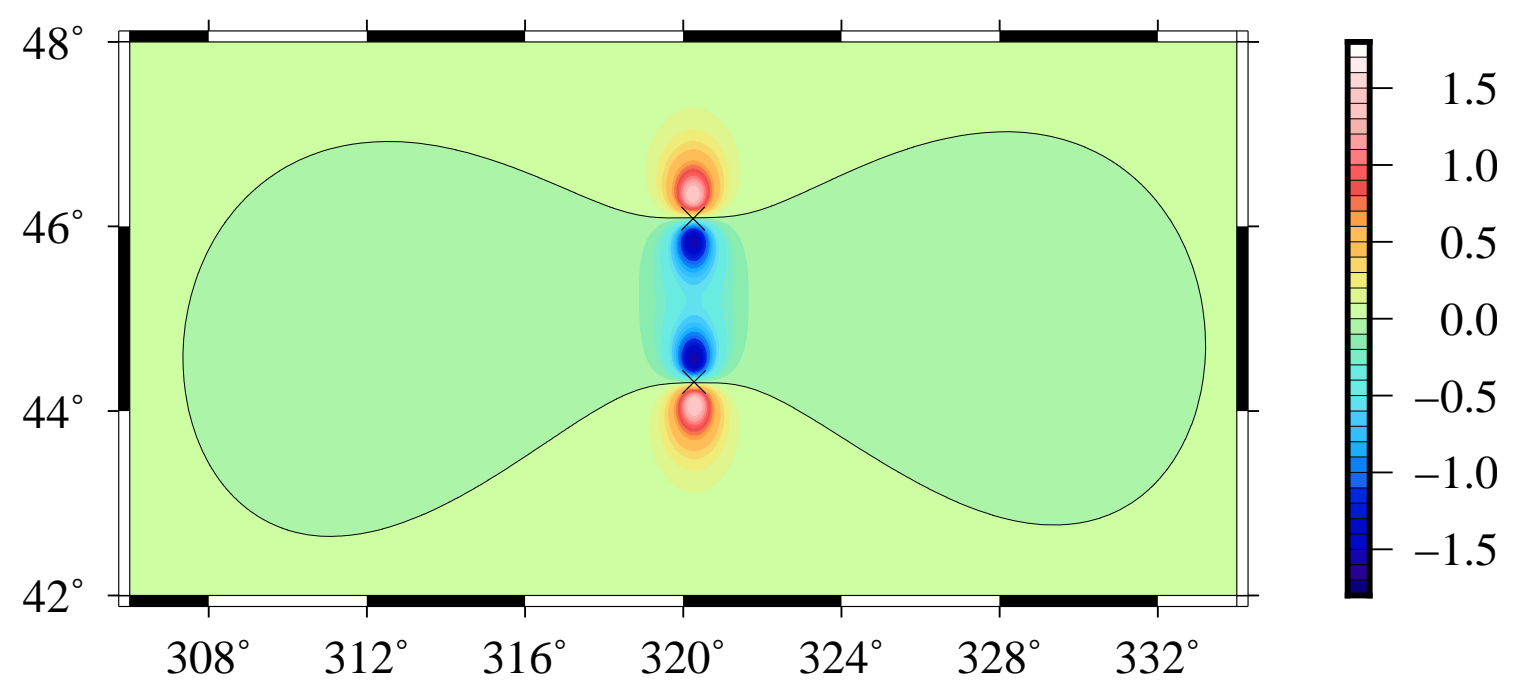

Figure 2: Spatial behaviour of the sub-integral kernel $\delta\left[\nabla \mathcal{H}_{0}\right] \cdot \mathbf{b}$. The kernel values are dimensionless as they were divided by the radius of the Brillouin sphere $R=1738528 \mathrm{~m}$. Positions of Ebb and Flow are shown by the lower and upper crosses. The black solid line indicates the zero values of the sub-integral kernel function.

\subsection{Spherical harmonic representation of truncation errors}

The BVP-based integral transformation of Eq. (23) assumes that the radial derivatives of the disturbing gravitational potential are known continuously over the whole Brillouin sphere (i.e., the full spatial angle $\Omega^{\prime}$ ). In practical calculations, however, the input data cover only limited geographical areas, which leads to a truncated integration (e.g., Molodensky et al., 1962; Novák et al., 2001) and hence a truncation error. Spatially restricted integrals are nevertheless favourable for solving inverse problems because they are numerically more stable (e.g., Tenzer and Novák, 2008). Accurate gravitational field modelling by truncated integration requires quantifying this neglected far-zone part, i.e., the truncation error. In this section, we derive efficient formulas for the numerical evaluation of the truncation errors from global GFM.

The truncated integration at the computation point is performed in the domain $\Omega_{0}^{\prime}$ defined by $\alpha \in\left[0^{\circ}, 180^{\circ}\right]$ and $\psi \in\left[0^{\circ}, \psi_{0}\right]$, where $\psi_{0}$ is the size of a spherical cap surrounding each computation point. It results in the contribution of the near zones. The integration in the complementary domain $\Omega^{\prime}-\Omega_{0}^{\prime}$, with $\alpha \in\left[0^{\circ}, 180^{\circ}\right]$ and $\psi \in\left(\psi_{0}, 180^{\circ}\right]$, represents the truncation error or the effect of the far zones.

The situation becomes more complex for the LoS disturbing gravitational acceleration via Eq. (23), as follows. Evaluation of this functional involves two GRAIL satellites and thus two computation points. Therefore, the same-sized spherical caps centred on Ebb and Flow specify two (overlapping) domains $\Omega_{1}^{\prime}$ and $\Omega_{2}^{\prime}$ and their far-zone complements $\Omega^{\prime}-\Omega_{1}^{\prime}$ and $\Omega^{\prime}-\Omega_{2}^{\prime}$. This leads to the spatial (integral) representation of the truncation error for the integral transformation of Eq. (23) in the form:

$$
\begin{aligned}
& \{\delta[\nabla T(r, \Omega)] \cdot \mathbf{b}\} \begin{array}{c}
\Omega^{\prime}-\Omega_{1}^{\prime} \\
\Omega^{\prime}-\Omega_{2}^{\prime}
\end{array} \\
& =(-1)^{k} \frac{R^{k-1}}{4 \pi}\left\{\int_{\Omega^{\prime}-\Omega_{2}^{\prime}} \mathcal{T}^{k}\left(R, \Omega^{\prime}\right)\left[b_{r_{2}} \mathcal{H}_{k}^{t}\left(t_{2}, u_{2}\right)+\left(b_{\varphi_{2}} \cos \alpha_{2}-b_{\lambda_{2}} \sin \alpha_{2}\right) \mathcal{H}_{k}^{u}\left(t_{2}, u_{2}\right)\right] \mathrm{d} \Omega^{\prime}\right.
\end{aligned}
$$




$$
\begin{aligned}
& \left.-\int_{\Omega^{\prime}-\Omega_{1}^{\prime}} \mathcal{T}^{k}\left(R, \Omega^{\prime}\right)\left[b_{r_{1}} \mathcal{H}_{k}^{t}\left(t_{1}, u_{1}\right)+\left(b_{\varphi_{1}} \cos \alpha_{1}-b_{\lambda_{1}} \sin \alpha_{1}\right) \mathcal{H}_{k}^{u}\left(t_{1}, u_{1}\right)\right] \mathrm{d} \Omega^{\prime}\right\} \\
& =(-1)^{k} \frac{R^{k-1}}{4 \pi} \int_{\Omega^{\prime}} \mathcal{T}^{k}\left(R, \Omega^{\prime}\right) \delta\left[b_{r} \Delta \mathcal{H}_{k}^{t}\left(t, u, u_{0}\right)\right. \\
& \left.+\left(b_{\varphi} \cos \alpha-b_{\lambda} \sin \alpha\right) \Delta \mathcal{H}_{k}^{u}\left(t, u, u_{0}\right)\right] \mathrm{d} \Omega^{\prime} .
\end{aligned}
$$

The two independent integrals after the first equality are performed over the far zones $\Omega^{\prime}-\Omega_{1}^{\prime}$ and $\Omega^{\prime}-\Omega_{2}^{\prime}$. The formulation in terms of the difference operator $\delta$ on the right-hand-side of Eq. (28) is possible by introducing the isotropic error kernels $\Delta \mathcal{H}_{k}^{t}$ and $\Delta \mathcal{H}_{k}^{u}$, when only one integral over the full spatial angle $\Omega^{\prime}$ is restored. The isotropic error kernels are:

$$
\begin{aligned}
& \Delta \mathcal{H}_{k}^{t}\left(t, u, u_{0}\right)=\left\{\begin{array}{ll}
0, & u_{0} \leq u \leq 1 \\
\mathcal{H}_{k}^{t}(t, u), & -1 \leq u<u_{0}
\end{array}=\sum_{n=0}^{\infty} \frac{2 n+1}{2} Q_{n}^{t, k}\left(t, u_{0}\right) P_{n, 0}(u),\right. \\
& \Delta \mathcal{H}_{k}^{u}\left(t, u, u_{0}\right)=\left\{\begin{array}{ll}
0, & u_{0} \leq u \leq 1 \\
\mathcal{H}_{k}^{u}(t, u), & -1 \leq u<u_{0}
\end{array}=\sum_{n=1}^{\infty} \frac{2 n+1}{2} Q_{n}^{u, k}\left(t, u_{0}\right) P_{n, 1}(u),\right.
\end{aligned}
$$

where $u_{0}=\cos \psi_{0}$. In the spatial form, these represent discontinuous functions that are identical to $\mathcal{H}_{k}^{t}$ and $\mathcal{H}_{k}^{u}$, except for $\psi \in\left[0^{\circ}, \psi_{0}\right]$ when they are zero. In the spectral form, the isotropic error kernels represent Legendre series (e.g., Hobson, 1965, Chap. 7) with the basis functions $P_{n, 0}$ and $P_{n, 1}$, see also Eqs. (24) and (25).

The coefficients $Q_{n}^{t, k}$ and $Q_{n}^{u, k}$ of these series expansions, also termed truncation error coefficients in the geodetic literature (e.g., Molodensky et al., 1962), are:

$$
\begin{aligned}
& Q_{n}^{t, k}\left(t, u_{0}\right)=\int_{-1}^{+1} \Delta \mathcal{H}_{k}^{t}\left(t, u, u_{0}\right) P_{n, 0}(u) \mathrm{d} u=\int_{-1}^{u_{0}} \mathcal{H}_{k}^{t}(t, u) P_{n, 0}(u) \mathrm{d} u \\
& =-t^{2} \frac{\partial}{\partial t} Q_{n}^{k}\left(t, u_{0}\right), \quad n \geq 0 \\
& Q_{n}^{u, k}\left(t, u_{0}\right)=\frac{1}{n(n+1)} \int_{-1}^{+1} \Delta \mathcal{H}_{k}^{u}\left(t, u, u_{0}\right) P_{n, 1}(u) \mathrm{d} u=\frac{1}{n(n+1)} \int_{-1}^{u_{0}} \mathcal{H}_{k}^{u}(t, u) P_{n, 1}(u) \mathrm{d} u \\
& =t\left[\frac{\sqrt{1-u_{0}^{2}}}{n(n+1)} \mathcal{H}_{k}\left(t, u_{0}\right) P_{n, 1}\left(u_{0}\right)+Q_{n}^{k}\left(t, u_{0}\right)\right], \quad n>0 .
\end{aligned}
$$

Equation (31) follows from the orthogonality relations $\int_{-1}^{+1} P_{n, 0}(u) P_{m, 0}(u) \mathrm{d} u=\frac{2}{2 n+1}$ and the definition of the isotropic kernel $\mathcal{H}_{k}^{t}$ by Eq. (24). The formula (32) results from an equivalent orthogonality relation $\int_{-1}^{+1} P_{n, 1}(u) P_{m, 1}(u) \mathrm{d} u=\frac{2 n(n+1)}{2 n+1}$ together with Eq. (25) for the isotropic kernel $\mathcal{H}_{k}^{u}$ and integration by parts.

The right-hand-sides of Eqs. (31) and (32) show that $Q_{n}^{t, k}$ and $Q_{n}^{u, k}$ are related to another set of coefficients $Q_{n}^{k}$. In other words, the problem of determining $Q_{n}^{t, k}$ and $Q_{n}^{u, k}$ reduces to finding a formulation only for $Q_{n}^{k}$. This simplifies practical calculations because the truncation error coefficients $Q_{n}^{k}$ correspond to the original analytical solutions of the radial BVP, see Eq. (12). 
With the help of the isotropic kernel $\mathcal{H}_{k}$ defined by Eq. (13), we obtain:

$Q_{n}^{k}\left(t, u_{0}\right)=\int_{-1}^{u_{0}} \mathcal{H}_{k}(t, u) P_{n, 0}(u) \mathrm{d} u=\sum_{m=0}^{\infty} t^{m+1} \frac{(2 m+1) m !}{(m+k) !} e_{n, m}\left(u_{0}\right), \quad n \geq 0$.

The functions $e_{n, m}=\int_{-1}^{u_{0}} P_{n, 0}(u) P_{m, 0}(u) \mathrm{d} u$ are known as Paul's coefficients (Paul, 1973).

Based on the type of isotropic kernel (spectral or spatial), we distinguish two approaches for calculating $Q_{n}^{k}, Q_{n}^{t, k}$, and $Q_{n}^{u, k}$. In case of the spectral isotropic kernels, we directly exploit the right-hand-sides of Eq. (31)-(33) within the limited spectral band $m \in\left[N_{\min }, N_{\max }\right]$. For the spatial isotropic kernels, the coefficients $Q_{n}^{t, k}$ and $Q_{n}^{u, k}$ can be determined from the recursions of $Q_{n}^{k}$. Recurrences for $Q_{n}^{0}$, i.e., for the Abel-Poison integral, can be found in (Pavlis, 1991). In addition, Jekeli (1979) and Pavlis (1991) derived recurrence relations for the truncation error coefficients $Q_{n}^{1}$, i.e., for the Hotine (1969) integral. Truncation error coefficients for $Q_{n}^{k}$ with $k \geq 2$ can be obtained by the procedure described in (Sünkel, 1981).

Having now defined the isotropic error kernels and the truncation error coefficients, we can find the spherical harmonic expansion of the truncation error. First, we insert the spectral forms of $\Delta \mathcal{H}_{k}^{t}$ and $\Delta \mathcal{H}_{k}^{u}$ by Eqs. (29) and (30) into the last integral of Eq. (28). Next, assuming absolute convergence of these series, we change the order of integrations and summations. This operation gives three global integrals (e.g., Heiskanen and Moritz, 1967; Hagiwara, 1972; Thalhammer, 1994):

$\frac{2 n+1}{4 \pi} \int_{\Omega^{\prime}} \mathcal{T}^{k}\left(R, \Omega^{\prime}\right) P_{n, 0}(u) \mathrm{d} \Omega^{\prime}=\mathcal{T}_{n}^{k}(R, \Omega)$,
$\frac{2 n+1}{4 \pi} \int_{\Omega^{\prime}} \mathcal{T}^{k}\left(R, \Omega^{\prime}\right) P_{n, 1}(u) \cos \alpha \mathrm{d} \Omega^{\prime}=\frac{\partial \mathcal{T}_{n}^{k}(R, \Omega)}{\partial \varphi}$,
$\frac{2 n+1}{4 \pi} \int_{\Omega^{\prime}} \mathcal{T}^{k}\left(R, \Omega^{\prime}\right) P_{n, 1}(u) \sin \alpha \mathrm{d} \Omega^{\prime}=\frac{1}{\cos \varphi} \frac{\partial \mathcal{T}_{n}^{k}(R, \Omega)}{\partial \lambda}$.

Finally, by exploiting the right-hand-sides of Eqs. (34)-(36), we arrive at the expansion:

$$
\begin{aligned}
& \{\delta[\nabla T(r, \Omega)] \cdot \mathbf{b}\}_{\substack{\Omega^{\prime}-\Omega_{1}^{\prime} \\
\Omega^{\prime}-\Omega_{2}^{\prime}}}=(-1)^{k} \frac{R^{k-1}}{2} \delta\left[b_{r} \sum_{n=0}^{\infty} Q_{n}^{t, k}\left(t, u_{0}\right) \mathcal{T}_{n}^{k}(R, \Omega)\right. \\
& \left.+b_{\varphi} \sum_{n=1}^{\infty} Q_{n}^{u, k}\left(t, u_{0}\right) \frac{\partial}{\partial \varphi} \mathcal{T}_{n}^{k}(R, \Omega)-b_{\lambda} \sum_{n=1}^{\infty} Q_{n}^{u, k}\left(t, u_{0}\right) \frac{1}{\cos \varphi} \frac{\partial}{\partial \lambda} \mathcal{T}_{n}^{k}(R, \Omega)\right] .
\end{aligned}
$$

To understand some basic properties of the truncation error by Eq. (37), we investigated the behaviour of the coefficients $Q_{n}^{t, 0}$ and $Q_{n}^{u, 0}$. These were computed for the spatial isotropic kernels $\mathcal{H}_{0}^{t}$ and $\mathcal{H}_{0}^{u}$ of Eqs. (A.5) and (A.11) with $R=1738528 \mathrm{~m}$ and $r=1753600 \mathrm{~m}$. The spherical radius $R$ defines the lunar Brillouin sphere employed in this article, see Sect. 3. The spherical radius $r$ corresponds to the satellite altitude of $\sim 15 \mathrm{~km}$ above the Brillouin sphere, in consistency with the investigations of the sub-integral kernel in Fig. 2. We also considered three integration radii $\psi_{0}=1^{\circ}, 5^{\circ}$, and $10^{\circ}$.

Examples of the truncation error coefficients as a function of spherical harmonic degree $n \in[0,360]$ are illustrated in Fig. 3 . The magnitudes of the coefficients are largest for low degrees and thus affect the size of the truncation error most. By increasing the spherical harmonic degree, the coefficient values oscillate around zero (and tend to zero for $n \rightarrow \infty$ ). The magnitudes of $Q_{n}^{t, 0}$ and $Q_{n}^{u, 0}$, and therefore the size of the truncation error, are more pronounced for smaller 

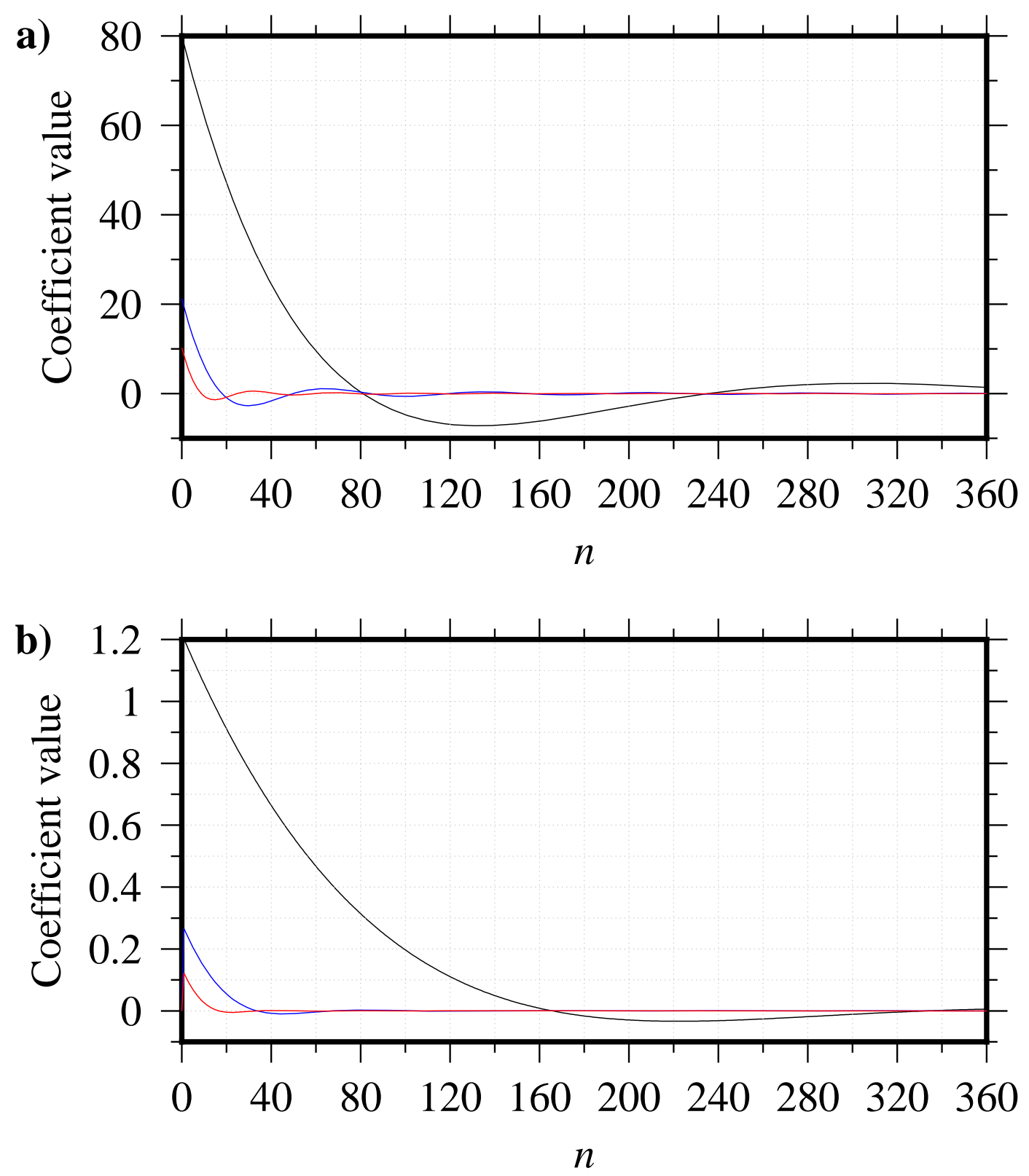

Figure 3: Truncation error coefficients: a) $Q_{n}^{t, 0}$ and b) $Q_{n}^{u, 0}$ for the integration radii $\psi_{0}=1^{\circ}$ (black), $5^{\circ}$ (blue), and $10^{\circ}$ (red).

integration radii. Another parameter affecting the amplitudes of the truncation error coefficients is the spherical radius $r$ or, equivalently, the satellite altitude, which exemplifies the complexity of the truncation error synthesis by Eq. (37).

\subsection{Integral estimator linearisation}

We now establish a linear mathematical model between the LoS disturbing gravitational acceleration and the radial derivatives of the disturbing gravitational potential. For this purpose, we initially formulate the remove-compute-restore (RCR) estimator. In general, this estimator decomposes any integral transformation in both the spatial and the spectral domains (e.g., Rapp and Rummel, 1975; Sansò and Sideris, 2013).

The spatial decomposition means splitting the integral transformation of Eq. (23) into the effects of the near zones and the truncation errors, see Sect. 2.2. The spectral decomposition 
divides the infinite spherical harmonic spectrum of the boundary condition $\mathcal{T}^{k}$, see Eqs. (10) and (11), into two parts: 1) the low-frequency part $n \in[0, N]$ and 2) the high-frequency part $n \in[N+1, \infty)$, where $N$ is the maximum spherical harmonic degree of the low-frequency part. These two operations provide the LoS disturbing gravitational acceleration in the following form:

$$
\begin{aligned}
& \delta[\nabla T(r, \Omega)] \cdot \mathbf{b} \\
& =(-1)^{k} \frac{R^{k-1}}{4 \pi}\left\{\int_{\Omega_{2}^{\prime}} \sum_{n=N+1}^{\infty} \mathcal{T}_{n}^{k}\left(R, \Omega^{\prime}\right)\left[b_{r_{2}} \mathcal{H}_{k}^{t}\left(t_{2}, u_{2}\right)+\left(b_{\varphi_{2}} \cos \alpha_{2}-b_{\lambda_{2}} \sin \alpha_{2}\right) \mathcal{H}_{k}^{u}\left(t_{2}, u_{2}\right)\right] \mathrm{d} \Omega^{\prime}\right. \\
& \left.-\int_{\Omega_{1}^{\prime}} \sum_{n=N+1}^{\infty} \mathcal{T}_{n}^{k}\left(R, \Omega^{\prime}\right)\left[b_{r_{1}} \mathcal{H}_{k}^{t}\left(t_{1}, u_{1}\right)+\left(b_{\varphi_{1}} \cos \alpha_{1}-b_{\lambda_{1}} \sin \alpha_{1}\right) \mathcal{H}_{k}^{u}\left(t_{1}, u_{1}\right)\right] \mathrm{d} \Omega^{\prime}\right\} \\
& +\frac{1}{R} \delta\left[-b_{r} \sum_{n=0}^{N} t^{n+2}(n+1) T_{n}(R, \Omega)+b_{\varphi} \sum_{n=1}^{N} t^{n+2} \frac{\partial}{\partial \varphi} T_{n}(R, \Omega)\right. \\
& \left.-b_{\lambda} \sum_{n=1}^{N} t^{n+2} \frac{1}{\cos \varphi} \frac{\partial}{\partial \lambda} T_{n}(R, \Omega)\right] \\
& +(-1)^{k} \frac{R^{k-1}}{2} \delta\left[b_{r} \sum_{n=N+1}^{\infty} Q_{n}^{t, k}\left(t, u_{0}\right) \mathcal{T}_{n}^{k}(R, \Omega)+b_{\varphi} \sum_{n=N+1}^{\infty} Q_{n}^{u, k}\left(t, u_{0}\right) \frac{\partial}{\partial \varphi} \mathcal{T}_{n}^{k}(R, \Omega)\right. \\
& \left.-b_{\lambda} \sum_{n=N+1}^{\infty} Q_{n}^{u, k}\left(t, u_{0}\right) \frac{1}{\cos \varphi} \frac{\partial}{\partial \lambda} \mathcal{T}_{n}^{k}(R, \Omega)\right] \cdot
\end{aligned}
$$

Equation (38) represents a complicated expression that originates from the general character of the LoS disturbing gravitational acceleration. When neglecting the difference operator $\delta$, it degenerates into:

- the negative gravity disturbance (for $b_{r}=1, b_{\varphi}=b_{\lambda}=0$ ),

- the South-North component of the vertical deflection (for $b_{\varphi}=1, b_{r}=b_{\lambda}=0$ ),

- the East-West component of the vertical deflection (for $b_{\lambda}=1, b_{r}=b_{\varphi}=0$ ),

- the total vertical deflection vector (for $b_{\varphi}=b_{\lambda}=1, b_{r}=0$ ),

- the total disturbance gravity vector (for $b_{r}=b_{\varphi}=b_{\lambda}=1$ ).

Thus, the mathematical apparatus in this article envelopes not only satellite observations, but also geodetic quantities collected by gravimetric, astronomic, and positioning instruments (e.g., Torge, 1989; Grafarend et al., 2006; Forsberg and Olesen, 2010; Timmen, 2010; Torge and Müller, 2012).

Being mathematically equivalent with the integral transformation of Eq. (23), the RCR estimator of Eq. (38) comprises three different terms. These are distinguished by the three pairs of large brackets. The first (integral) term on the right-hand-side represents the highfrequency part of the near zone LoS disturbing gravitational acceleration. The second (spherical harmonic synthesis) term is the low-frequency LoS disturbing gravitational acceleration. The 
third (spherical harmonic synthesis) term is the truncation error in the spectral band $n \in$ $[N+1, \infty)$, see Eq. (37). We acknowledge that numerous alternatives to the RCR estimator of Eq. (38) can be derived by modifying the isotropic kernels $\mathcal{H}_{k}^{t}$ and $\mathcal{H}_{k}^{u}$ (e.g., Jekeli, 1981; Vaníček and Featherstone, 1998; Evans and Featherstone, 2000; Sjöberg, 2003; Sjöberg and Featherstone, 2004; Šprlák, 2010).

The above three-term separation leads to the desired linear model (e.g., Grafarend, 2006, Sect. 2.1):

$\mathbf{l}=\mathbf{y}-\mathbf{y}_{1}-\mathbf{y}_{2}^{k}=\mathbf{A}_{k} \mathbf{x}^{k}+\boldsymbol{\epsilon}$.

The vector $\mathbf{x}^{k}$ substitutes the values of the radial derivatives of the disturbing gravitational potential. The design matrix $\mathbf{A}_{k}$ is composed of the sub-integral kernel values, including the term in front of the curly bracket in Eq. (38). The symbol $\boldsymbol{\epsilon}$ stands for the error vector and we assume that the errors are Gaussian with zero mean.

The observation vector $\mathbf{l}$ on the left-hand-side of Eq. (39) is determined by the LoS disturbing gravitational acceleration vector $\mathbf{y}$, which is reduced by its low-frequency and the high-frequency truncation error counterparts $\mathbf{y}_{1}$ and $\mathbf{y}_{2}^{k}$. This subtraction is possible because the vectors $\mathbf{y}_{1}$ and $\mathbf{y}_{2}$ can be synthesised from an already-available global GFM and thus are a priori known. In practical computations, the vector $\mathbf{y}_{2}^{k}$, i.e., the high-frequency truncation error is neglected. We emphasise, however, that this is only permitted by "optimally" selecting the size of the spherical cap $\psi_{0}$ and the maximum spherical harmonic degree of the low-frequency part $N$. On Earth, this is often done by comparisons with GPS-levelling data, so the "optimisation" is really only empirical.

\section{Numerical experiments}

In this Section, we perform synthetic tests of the upward continuation (direct problem), i.e., we validate the integral transformation of Eq. (23) and investigate the accuracy of the RCR estimator by Eq. (38). Next, we assess the performance of the downward continuation (inverse problem) and invert actual GRAIL observations to obtain high-resolution regional GFMs. In this presentation, we restrict the numerical experiments to $k=0$ and 1 for brevity. The corresponding quantities, i.e., the disturbing gravitational potential $\mathcal{T}^{0}$ and its first radial derivative $\mathcal{T}^{1}$, are used in regional studies (e.g., Sugano and Heki, 2004; Goossens et al., 2014; Tenzer et al., 2018).

Throughout these numerical experiments, the normal gravitational field of the Moon was generated by an equipotential spheroid. It is defined by four parameters: the major semi-axis $a$ $=1737325.0 \mathrm{~m}$, the minor semi-axis $b=1736789.0 \mathrm{~m}$, the planetocentric gravitational constant $G M=4902.8 \times 10^{9} \mathrm{~m}^{3} \mathrm{~s}^{-2}$, and the angular velocity $\omega=2.6617 \times 10^{-6} \mathrm{rad} \mathrm{s}^{-1}$ as determined by Ardalan and Karimi (2014).

The LoS disturbing gravitational accelerations depend on the positions of the two GRAIL satellites and are irregular double-point values. On the other hand, values of $\mathcal{T}^{0}$ and $\mathcal{T}^{1}$ are located at the centres of the integration elements and form equiangular grids on the Brillouin sphere. We have strictly followed the notion of the Brillouin sphere, because our mathematical apparatus is based upon the analytical solution of Laplace's equation, see Sect. 1.2.

The numerical experiments were carried out in the north-western part of the nearside of the Moon. The area covers the Montes Jura mountain range, see Fig. 4c. We distinguished between the computational area (bounded by $\varphi \in\left[40^{\circ}, 50^{\circ}\right]$ and $\lambda \in\left[-45^{\circ},-35^{\circ}\right]$ ) and data areas (extending each side of the computational area by integration radii). The characteristics of the topography and of the gravitational field are summarised by descriptive statistics in Tab. 1 and are mapped in Figs. 4, 5a and 5b. The adopted radius of the Brillouin sphere $R=1738528$ $\mathrm{m}$ was determined from the maximum topographic height of $1128 \mathrm{~m}$ within the computational area. 


\begin{tabular}{c|c|c|cccc}
\hline Quantity & Unit & Discretisation & Min. & Max. & Mean & Std. dev. \\
\hline $\mathcal{T}^{0}$ & $\mathrm{~m}^{2} \mathrm{~s}^{-2}$ & $9 / 70^{\circ}$ & -8.464 & 5.376 & 0.009 & 1.496 \\
$\mathcal{T}^{1}$ & $\mathrm{mGal}$ & $9 / 70^{\circ}$ & -106.869 & 123.443 & -0.175 & 23.945 \\
$H$ & $\mathrm{~m}$ & $9 / 140^{\circ}$ & -4756 & 1128 & -1495 & 864 \\
\hline
\end{tabular}

Table 1: Statistics of $\mathcal{T}^{0}, \mathcal{T}^{1}$, and $H$ (spherical height referred to the sphere of radius $\left.1737400 \mathrm{~m}\right)$ in the computation area. $\mathcal{T}^{0}$ and $\mathcal{T}^{1}$ were synthesised between degrees 200-700 from GL1500E (Konopliv et al., 2014) on the Brillouin sphere $(R=1738528 \mathrm{~m})$.

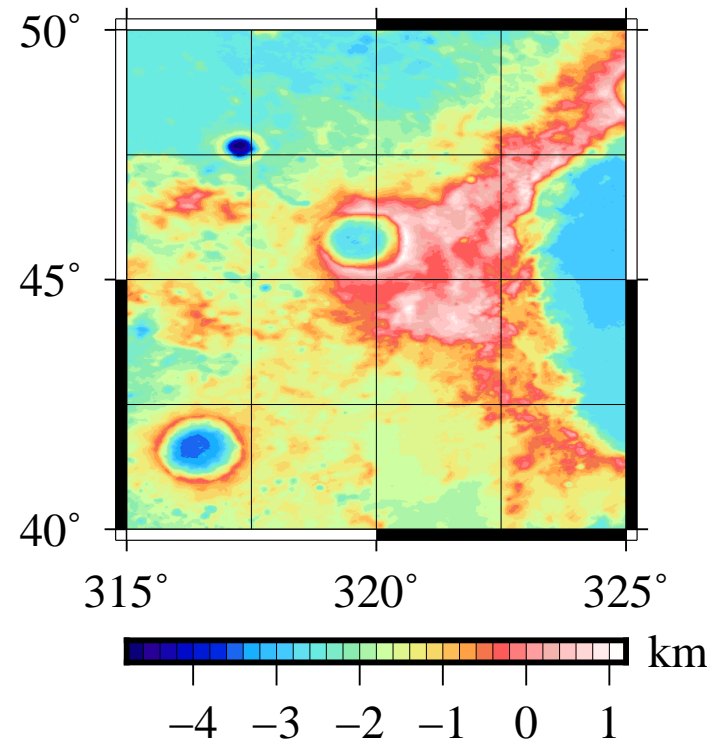

Figure 4: Map of LOLA topographic heights in the computation area.

\subsection{Synthetic tests on the direct problem}

\subsubsection{Integral transformation}

In the first numerical experiment, we checked the correctness of the integral formula by Eq. (23). Namely, we numerically tested the upward continuation of $\mathcal{T}^{0}$ and $\mathcal{T}^{1}$ onto the LoS disturbing gravitational acceleration.

First, we synthesised equiangular global grids of $\mathcal{T}^{0}$ and $\mathcal{T}^{1}$ from the lunar global GFM GL1500E (Konopliv et al., 2014) on the surface of the Brillouin sphere for the computation area $(R=1738528 \mathrm{~m})$. We performed the spherical harmonic synthesis between spherical harmonic degrees 200-700. We restricted the upper bound of the spectral band, because GL1500E gravitational field is dominated by noise beyond degree $~ 700$ (e.g., Konopliv et al., 2014; Lemoine et al., 2014), see below. The regular grids were calculated with the discretisations of $9 / 35^{\circ}$ and $9 / 70^{\circ}$. The former discretisation corresponds to the maximum spatial resolution at degree $n$ $=700$, while the latter discretisation represents a geometric refinement by one half. The statistics of the equiangular global grids are in Tab. 2.

Second, the LoS disturbing gravitational acceleration was computed from GL1500E (Kono- 


\begin{tabular}{c|c|c|cccc}
\hline Quantity (Mission) & Unit & Discretisation & Min. & Max. & Mean & Std. dev. \\
\hline $\mathcal{T}^{0}$ & $\mathrm{~m}^{2} \mathrm{~s}^{-2}$ & $9 / 35^{\circ}$ & -46.613 & 61.002 & 0.000 & 2.883 \\
& & $9 / 70^{\circ}$ & -55.715 & 58.879 & 0.000 & 2.883 \\
$\mathcal{T}^{1}$ & \multirow{3}{*}{$\mathrm{mGal}$} & $9 / 35^{\circ}$ & -1425.295 & 1286.996 & -0.026 & 50.779 \\
& & $9 / 70^{\circ}$ & -1376.300 & 1567.109 & -0.026 & 50.779 \\
$\delta[\nabla T(r, \Omega)] \cdot \mathbf{b}(\mathrm{PM})$ & $\mathrm{mGal}$ & - & -868.288 & 712.334 & 0.345 & 95.104 \\
$\delta[\nabla T(r, \Omega)] \cdot \mathbf{b}(\mathrm{XM} 2)$ & $\mathrm{mGal}$ & - & -6852.993 & 6370.529 & 7.444 & 1329.310 \\
\hline
\end{tabular}

Table 2: Statistics of the equiangular global grids of $\mathcal{T}^{0}$ and $\mathcal{T}^{1}$ (evaluated globally), and of the LoS disturbing gravitational acceleration $\delta[\nabla T(r, \Omega)] \cdot \mathbf{b}$ (evaluated in the computation area).

pliv et al., 2014) in the spectral band $n=200-700$ at the positions of Ebb and Flow. Their orbits are available at the GRAIL SPICE data archive, see https://naif.jpl.nasa.gov/pub/ naif/pds/data/grail-1-spice-6-v1.0/grlsp_1000/. We considered only satellite locations within the computational area formed by two orbital datasets: 1) 3696 points of the GRAIL PM, and 2) 3251 points of the GRAIL EM between the period from 27 October 2012 until 14 December 2012 (XM2). The statistics of the two LoS datasets are listed in Tab. 2.

These two datasets differ significantly by the average altitudes of Ebb and Flow above the Brillouin sphere. The average altitudes are $\sim 54 \mathrm{~km}$ for PM and $\sim 21 \mathrm{~km}$ for XM2, and span from $\sim 13 \mathrm{~km}$ to $\sim 88 \mathrm{~km}$. This allowed us to test the integral transformation of Eq. (23) for quite different geometric/orbital configurations. Orbits from the GRAIL EM between the period from 30 August 2012 until 26 October 2012 (XM1) were not taken into account in the simulation, because these represent an intermediate geometric scenario between PM and XM2.

Thirdly, the global equiangular grids of $\mathcal{T}^{0}$ and $\mathcal{T}^{1}$ were upward continued by the integral transformation of Eq. (23). This upward continuation was performed with the spatial forms of the isotropic kernels by Eqs. (A.5), (A.6), (A.11), and (A.12).

\begin{tabular}{c|c|c|cccc}
\hline Mission & Unit & Discretisation & Min. & Max. & Mean & Std. dev. \\
\hline \multirow{2}{*}{$\mathrm{PM}$} & $\mu \mathrm{Gal}$ & $9 / 35^{\circ}$ & -0.015 & 0.016 & 0.000 & 0.002 \\
& $\mathrm{nGal}$ & $9 / 70^{\circ}$ & 0.003 & 0.022 & 0.008 & 0.004 \\
\hline \multirow{2}{*}{$\mathrm{XM} 2$} & $\mu \mathrm{Gal}$ & $9 / 35^{\circ}$ & -55.687 & 52.325 & -0.011 & 5.402 \\
& $\mathrm{nGal}$ & $9 / 70^{\circ}$ & -2.294 & 1.771 & 0.002 & 0.173 \\
\hline
\end{tabular}

Table 3: Statistics of the differences between the LoS disturbing gravitational acceleration computed by the numerical integration of $\mathcal{T}^{0}$ and by spherical harmonic synthesis.

Finally, we compared the values of the LoS disturbing gravitational acceleration computed by numerical integration with those from spherical harmonic synthesis. Descriptive statistics of the differences are presented in Table 3 when integrating over the global grids of $\mathcal{T}^{0}$. Similar statistical values were also obtained when continuing $\mathcal{T}^{1}$, but are not presented in Table 3 as the conclusions remain the same.

The numerical performance of the upward continuation differs among the two datasets. The PM dataset (with higher average altitude) is characterised by smaller standard deviations as compared to the results for the XM2 (with much lower satellite altitudes). The distinction originates from the behaviour of the sub-integral kernel, see Fig. 2. This kernel function possesses a high spatial gradient towards Ebb and Flow located at low orbits, while it is smoother for increasing altitudes of the GRAIL satellites. Numerical evaluation of the integral transformation 
of Eq. (23) performs better for smoother functions.

Approximation of the sub-integral kernel can be enhanced by refining the spatial resolution of equiangular grids; see Tab. 3. This may be applied when solving the direct problem, e.g., by a finer sampling of the original grid. For the inverse problem, however, the higher spatial resolution leads to more unknowns. This, in particular, is undesirable for the GRAIL mission, because the number of observations is limited and may be even less than twice the amount of unknowns for the discretisation of $9 / 70^{\circ}$, see Sects. 3.2 and 3.3, leading to underdetermined or poorly conditioned normal equations.

To exploit the integral formula in Eq. (23) for calculations with real data, its performance has to be confronted with simulated observation errors. The noise of the GRAIL LoS gravitational acceleration may be defined by the power spectral density (PSD) $Q$. The mean values of $Q$ are $\sim 4 \mu \mathrm{Gal}^{2} \mathrm{~Hz}^{-1}$ (for the PM) (Han, 2013) and $\sim 100 \mu \mathrm{Gal}^{2} \mathrm{~Hz}^{-1}$ (for EM). Assuming the noise is Gaussian with zero mean, $Q$ can be related to the noise standard deviation $\sigma$ and the data sampling in the time domain $\Delta t$ as follows:

$\sigma=\pi \sqrt{\frac{Q}{2 \Delta t}}$.

This last expression gives estimates of the noise standard deviations of $2 \mu \mathrm{Gal}$ (for the PM) and $15 \mu \mathrm{Gal}$ (for EM) for the corresponding data sampling rates of $5 \mathrm{~s}$ and $2 \mathrm{~s}$. As a rule of thumb, performance of a gravitational field modelling approach should be about an order better than the noise level. This requirement is fulfilled for the PM and XM2 datasets by employing the discretisation of $9 / 70^{\circ}$. Such spatial resolution is a trade-off between accurate approximation of the integral transformation of Eq. (23) and data redundancy and was therefore employed in the numerical experiments below.

\subsubsection{Synthetic remove-compute-restore test}

In this numerical experiment, we investigate the precision of the RCR estimator by Eq. (38). The spatially limited integration over the residual grids with the sampling of $9 / 70^{\circ}$ was carried out with the spherical cap $\psi_{0}=5^{\circ}$. The high-resolution truncation error was synthesised from GL1500E between degrees $n=200-700$ at the positions of Ebb and Flow independently for $\mathcal{T}^{0}$ and $\mathcal{T}^{1}$.

The contributions of the limited integration domain and of the truncation error were added and compared with their "true" counterparts from the global integration (Section 3.1.1). For both quantities $\mathcal{T}^{0}$ and $\mathcal{T}^{1}$, we found comparable standard deviations reaching $\sim 0.1 \mu \mathrm{Gal}$ for PM and $\sim 0.9 \mu \mathrm{Gal}$ for XM2. Thus, the corresponding RCR estimators are applicable for gravitational field modelling as they meet the performance requirement stated above.

Similar numerical experiments were repeated for smaller integration radii $\psi_{0}=1^{\circ}$ and $3^{\circ}$. For these, we observed a significant increase of the standard deviations exceeding the performance criterion. We attribute the degradation of the numerical accuracy to neglect of spherical harmonic terms beyond degree 700 when synthesising the truncation error, i.e., an omission error. This is indicated by the behaviour of the truncation error coefficients, see Fig. 3. For smaller integration radii, these oscillate slowly and possess much higher amplitudes. Consequently, higher-degree spherical harmonic terms are important when evaluating the truncation error.

\subsection{Synthetic inversion of LoS disturbing gravitational acceleration}

The purpose of this numerical experiment is to investigate the performance of the downward continuation for the synthetic lunar gravitational field. We calculated design matrices $\mathbf{A}_{k}$ by the spatial forms of the isotropic kernels with the integration radii $\psi_{0}=5^{\circ}\left(\right.$ for $\left.\mathcal{T}^{0}\right)$ and $7^{\circ}$ (for $\mathcal{T}^{1}$ ). We found these spherical cap sizes preferable, because the magnitudes of the high-frequency 
part $(N>199)$ of the truncation errors were below the performance requirement of GRAIL noise and thus may have been neglected.

The observation vectors 1 comprised 56111 and 80374 entries for $\mathcal{T}^{0}$ and $\mathcal{T}^{1}$, respectively. The unknown vectors $\mathbf{x}^{k}$ originated from discretising the Brillouin sphere by the spacing of $9 / 70^{\circ}$ in the data areas and were given by 32232 (for $k=0$ ) and 47188 (for $k=1$ ) values. The recovered grids of $\mathcal{T}^{0}$ and $\mathcal{T}^{1}$ were evaluated only in the smaller computational area. This limitation was necessary because estimates close to the boundaries of the data areas did not exactly fulfil the linear mathematical model (due the spherical caps extending beyond the data areas) and were affected by systematic errors.

Least-squares estimates of $\mathcal{T}^{0}$ and $\mathcal{T}^{1}$ can be found for the discretised overdetermined equation systems as:

$\hat{\mathbf{x}}^{k}=\left(\mathbf{A}_{k}^{T} \mathbf{P} \mathbf{A}_{k}\right)^{-1} \mathbf{A}_{k}^{T} \mathbf{P} \mathbf{l}$,

where $\mathbf{P}$ is the weight matrix. To prove this assertion, we exploited the design matrices specified above. In addition, we calculated $\mathbf{A}_{k}$ without considering any truncated spherical caps around the computation points (cf. Xu, 1998; Eshagh, 2011; Kingdon and Vaníček, 2011; Shen et al., 2012). We set the elements of the weight matrix to $(2 \mu \mathrm{Gal})^{-2}$ for the PM and $(15 \mu \mathrm{Gal})^{-2}$ for the EM throughout the numerical experiments.

We found that reasonable least-squares solutions could be obtained for the design matrices with the spherical caps, while the least-squares estimates were useless when neglecting cap sizes. We emphasise that the least-squares solutions were found for the non-perturbed observation vectors calculated by the upward continuation. Such poor performance can be explained by the condition numbers of the normal matrices $\mathbf{A}_{k}^{T} \mathbf{P} \mathbf{A}_{k}$. For the spherical cap scenario, the condition numbers reached $\sim 10^{10}$. This is five orders lower than the threshold of $\sim 10^{15}$ for the loss of all significant digits in double precision arithmetic. The normal matrices could be still inverted and the least-squares estimates appeared feasible. Without considering the cap sizes, however, the least-squares method completely failed, as the condition numbers were greater than $\sim 10^{20}$. Thus, considering a spherical cap may partially stabilise the system of normal equations.

In reality, the observation vector $\mathbf{l}$ is affected by perturbations, e.g., due to data noise or rounding errors. In addition, the linear mathematical model may not be exact, e.g., due to approximation errors. In such cases, we cannot find any reasonable least-squares estimate and the inverse problem is indeed ill-posed.

Numerous methods have been developed for solving ill-posed problems; see (e.g., Bouman, 1998; Hansen, 1998; Xu, 1998; Koch and Kusche, 2002; Xu et al., 2006; Xu, 2009; Freeden and Nashed, 2018) and the references therein. We employed Tikhonov-Phillips regularisation providing the estimate of $\mathbf{x}^{k}$ as (Phillips, 1962; Tikhonov, 1963a,b):

$\hat{\mathbf{x}}_{T}^{k}=\left(\mathbf{A}_{k}^{T} \mathbf{P} \mathbf{A}_{k}+\gamma^{2} \mathbf{I}\right)^{-1} \mathbf{A}_{k}^{T} \mathbf{P} \mathbf{l}$,

with $\gamma^{2}$ being the regularisation parameter. In general, regularised estimates are biased. We determined the regularisation bias (standard deviation of which was about an order lower than that of $\mathbf{x}^{k}$ throughout the numerical experiments) by the formula (Shen et al., 2012):

$\hat{\mathbf{b}}^{k}=-\gamma^{2}\left(\mathbf{A}_{k}^{T} \mathbf{P} \mathbf{A}_{k}+\gamma^{2} \mathbf{I}\right)^{-1} \hat{\mathbf{x}}_{T}^{k}$,

that led to the bias-corrected regularised estimates of the form:

$\hat{\mathbf{x}}_{B C}^{k}=\hat{\mathbf{x}}_{T}^{k}-\hat{\mathbf{b}}^{k}$.

Probably the most crucial parameter of the regularisation is $\gamma^{2}$, which was computed by the L-curve criterion (Miller, 1970). All regularisations were performed by the MATLAB package Regularisation Tools (Hansen, 2007), which factorises the design matrix by singular value 
decomposition.

The synthetic observation vectors 1 were represented by the LoS disturbing gravitational acceleration between degrees $n=200-700$ synthesised from GL1500E. The values of 1 were contaminated by Gaussian noise with zero mean and standard deviations of $2 \mu \mathrm{Gal}$ for the PM and $15 \mu \mathrm{Gal}$ for the EM. These white noise characteristics are close to the stochastic properties of the real GRAIL observations (e.g., Konopliv et al., 2014; Lemoine et al., 2014).

The statistics of $\mathbf{l}$ and of the white noise in the data area for $\mathcal{T}^{0}$ are presented in Table 4 . It can be seen that the extrema of the high-degree LoS disturbing gravitational accelerations reach several mGals for the EM and are smaller by about an order for the PM. The relative noise, i.e., the ratio of the standard deviation of the white noise to that of the signal, reaches $\sim 2.9 \%$ for $\mathrm{PM}, \sim 2.3 \%$ for XM1, and $\sim 1.5 \%$ for XM2.

\begin{tabular}{c|cccc}
\hline Quantity (Mission) & Min. & Max. & Mean & Std. dev. \\
\hline $\mathbf{l}(\mathrm{PM})$ & -868.288 & 755.462 & -0.008 & 68.128 \\
$\mathbf{l}(\mathrm{XM} 1)$ & -5296.752 & 6049.590 & -0.669 & 629.371 \\
$\mathbf{l}(\mathrm{XM} 2)$ & -7547.333 & 6989.405 & -0.730 & 964.769 \\
$\boldsymbol{\epsilon}(\mathrm{PM})$ & -7.544 & 7.424 & 0.011 & 1.995 \\
$\boldsymbol{\epsilon}(\mathrm{XM} 1)$ & -58.067 & 58.952 & -0.022 & 14.968 \\
$\boldsymbol{\epsilon}(\mathrm{XM} 2)$ & -62.396 & 59.714 & 0.132 & 14.952 \\
\hline
\end{tabular}

Table 4: Statistics (in $\mu \mathrm{Gal}$ ) of the observation vector $\mathbf{l}$ and the white noise in the data area for $\mathcal{T}^{0}$. The values of 1 were synthesised from GL1500E between degrees 200-700.

\begin{tabular}{c|c|cccc}
\hline Quantity & Unit & Min. & Max. & Mean & Std. dev. \\
\hline $\mathcal{T}^{0}$ & $\mathrm{~m}^{2} \mathrm{~s}^{-2}$ & -8.464 & 5.376 & 0.009 & 1.496 \\
$\Delta \mathcal{T}^{0}$ & & -1.686 & 1.403 & -0.021 & 0.363 \\
\hline $\mathcal{T}^{1}$ & \multirow{2}{*}{ mGal } & -106.869 & 123.443 & -0.175 & 23.945 \\
$\Delta \mathcal{T}^{1}$ & & -37.232 & 36.662 & 0.116 & 7.783 \\
\hline
\end{tabular}

Table 5: Statistics of the true values (synthesised from GL1500E) and the differences between the inversion estimates and the true values (designated by $\Delta \mathcal{T}^{k}, k=0,1$ ).

The statistics of the synthetic downward continuation in the computational area are summarised in Table 5. The disturbing gravitational potential and its first radial derivative can be recovered with accuracies reaching $0.363 \mathrm{~m}^{2} \mathrm{~s}^{-2}$ and $7.783 \mathrm{mGal}$. This corresponds to $24 \%$ and $33 \%$ of the respective signal variations. The maps of the true values, estimates and their differences are illustrated in Fig. 5. We observe that the shapes of all features are preserved within the computation area (red dashed box), proving the performance of the simulated inversion. The differences are higher beyond the computation area, some circular features, such as those with the centres at $\varphi=53^{\circ}, \lambda=-43.5^{\circ}$ or $\varphi=49^{\circ}, \lambda=-34.5^{\circ}$, could still be recovered.

\subsection{Inversion of GRAIL LoS disturbing gravitational acceleration}

The aim of this numerical experiment is the regional recovery of $\mathcal{T}^{0}$ and $\mathcal{T}^{1}$ from realistic GRAIL measurements. For the recovery of the static gravitational field, the real observables have to be corrected by non-conservative and time-variable forces. In addition, the LoS disturbing gravitational acceleration from the GRAIL DSN and LGRS data maybe significantly biased. 
a)

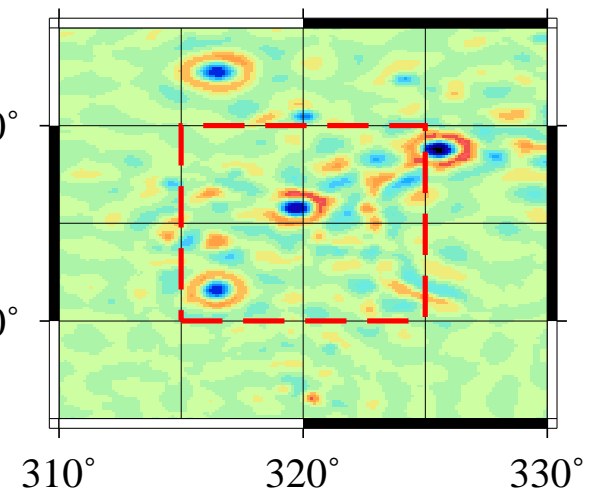

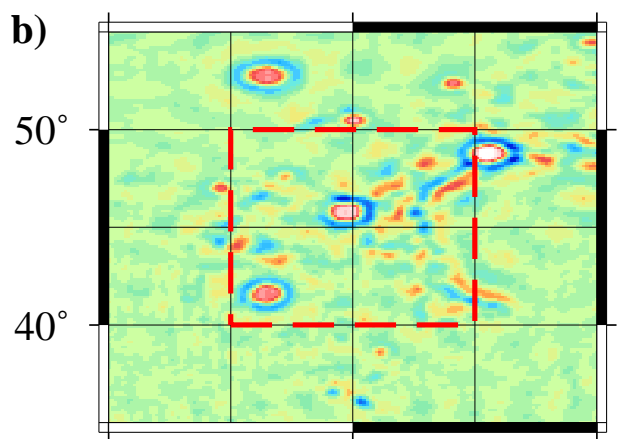

$310^{\circ} \quad 320^{\circ} \quad 330^{\circ}$

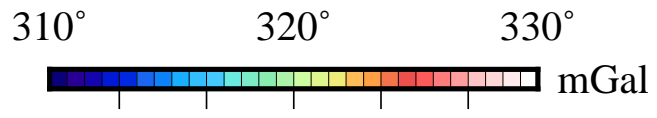

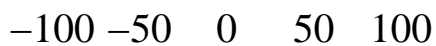

c)

$\begin{array}{lllll}-10 & -5 & 0 & 5 & 10\end{array}$

$50^{\circ}$

$40^{\circ}$

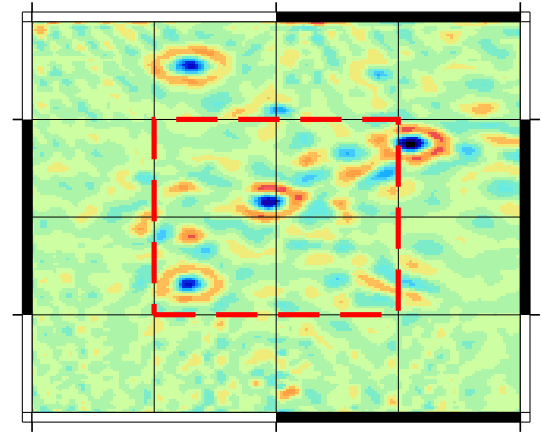

d)
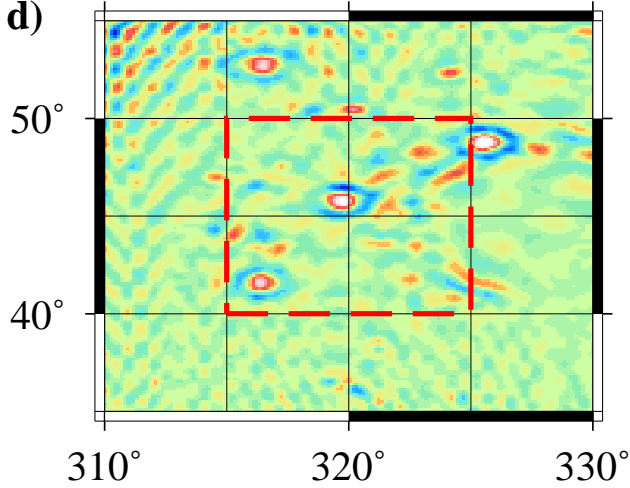
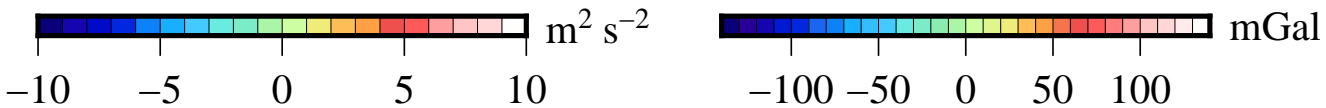

e)
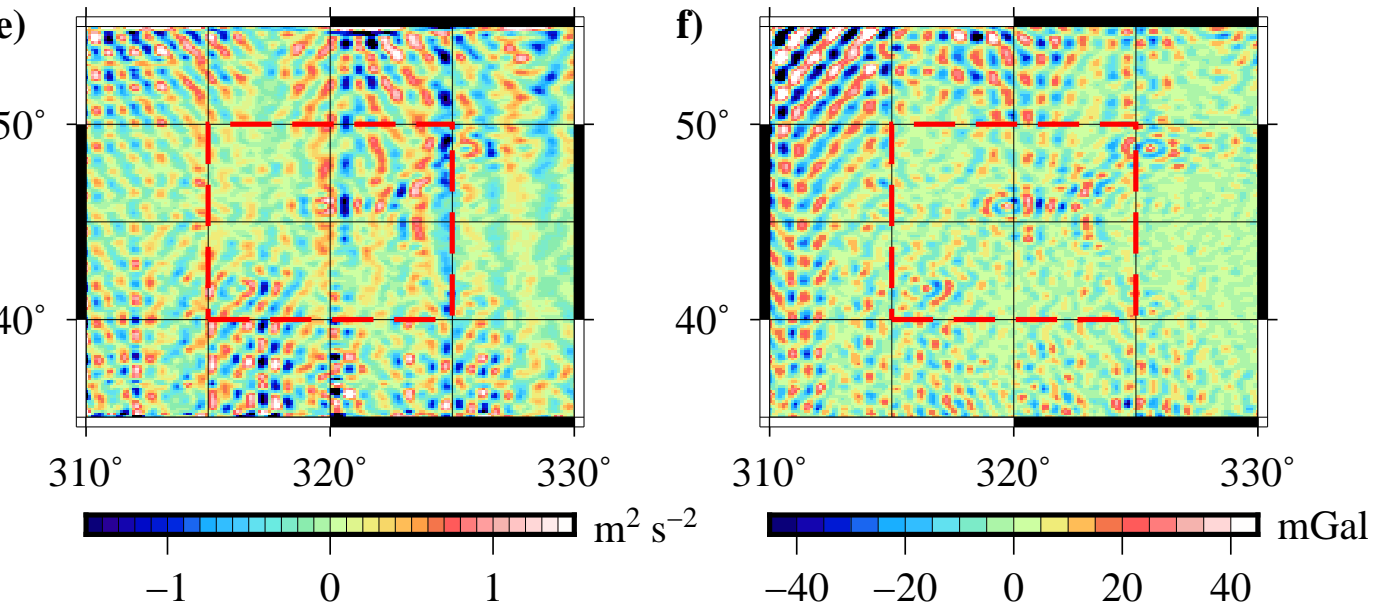

Figure 5: Maps of: a) $\mathcal{T}^{0}$ synthesised from GL1500E, b) $\mathcal{T}^{1}$ synthesised from GL1500E, c) $\mathcal{T}^{0}$ calculated by inversion, d) $\mathcal{T}^{1}$ calculated by inversion, e) $\Delta \mathcal{T}^{0}$, and f) $\Delta \mathcal{T}^{1}$. All quantities represent high-frequency part of the gravitational field between degrees 200-700. The red dashed line bounds the computation area.

The bias originates from the second term on the right-hand-side of Eq. (20), termed as the orbital correction term.

The non-conservative forces, the time-variable forces and the orbital correction term bias are prominent only in the low frequencies (e.g., Han, 2013). These effects could be removed by appropriate high-pass filtering of GRAIL LoS disturbing gravitational acceleration in the time 
domain. The cut-off frequency $f$ of the high-pass filter could be selected based on the maximum spherical harmonic degree of the low-frequency part $N$ of the RCR estimator, see Eq. (38). This is permitted by the approximate relationship between the frequency $f$ and the spherical harmonic degree $n$ (e.g., McKenzie and Nimmo, 1997):

$n \approx \frac{2 \pi f(R+H)}{v}$,

where $H$ is the average altitude of the twin satellites and $v$ is their average speed.

However, a gravitational signal available as a time series and filtered within the frequency band $f \in\left[f_{1}, f_{2}\right]$ does not match exactly with the gravitational signal in the spherical harmonic band $n \in\left[N_{1}, N_{2}\right]$, where $N_{1}$ and $N_{2}$ are calculated by Eq. (45). This inconsistency is investigated by Cai et al. (2013).

To address the characteristics of the GRAIL observables, we calculated the static LoS disturbing gravitational acceleration using the following procedure:

1. The GRAIL DSN and LGRS data were extracted from the SPICE archive and divided into daily arcs. We preserved only those daily time series when at least one sub-satellite point of the GRAIL twin satellites occurred in the data areas.

2. The preserved daily time series were combined to form continuous 2-7 days arc segments. The GRAIL LoS disturbing gravitational acceleration was calculated from the DSN and LGRS by Eq. (20).

3. Power spectral density was estimated by Welch's method for each segment and visually inspected. The segments were subdivided if the PSD reached greater values than the noise (i.e., $\sim 2 \mu \mathrm{Gal} \mathrm{Hz}{ }^{-1 / 2}$ for the $\mathrm{PM}$ and $\sim 15 \mu \mathrm{Gal} \mathrm{Hz}{ }^{-1 / 2}$ for EM), e.g., due to outliers or the angular momentum dump manoeuvres (e.g., Konopliv et al., 2013). In total, 36 arc sub-segments were formed: 15 for the PM and 21 for EM.

4. A high-pass Butterworth filter of 8th-degree was applied to the GRAIL LoS disturbing gravitational acceleration in each sub-segment. We selected the cut-off (minimum) frequency of $0.02 \mathrm{~Hz}$ of the high-pass filter, because the time-variable and conservative forces are below the noise level of GRAIL LoS gravitational acceleration beyond this frequency (Han, 2013).

5. The modelled LoS disturbing gravitational acceleration was calculated from GL1500E. The computation was performed at each of the locations of the 36 sub-segments by spherical harmonic synthesis; see the second term on the right-hand side in Eq. (38), between degrees $0-700$.

6. The modelled LoS disturbing gravitational acceleration in each sub-segment was low-pass filtered by an eighth-degree Butterworth filter with the cut-off (maximum) frequency of $0.02 \mathrm{~Hz}$. This low-pass filtering is complementary to the high-pass filtering in Step 4.

7. The corresponding high-pass and low-pass filtered LoS disturbing gravitational accelerations were added. In this way, we obtained combined full-band LoS disturbing gravitational accelerations and extracted only those within the data area.

8. To get the final GRAIL observation vector 1 , we reduced the combined full-band LoS gravitational accelerations in the data area by its low-frequency part. The low-degree spherical harmonic contribution was synthesised from GL1500E in the band $n=0-199$.

The performance of the combination procedure was validated by a closed-loop test. We synthesised the true LoS disturbing gravitational acceleration along all segments and applied the low-pass and high-pass Butterworth filters. The corresponding low-pass and high-pass filtered values were added and compared with the true ones. The differences reached at most $10^{-5} \mu \mathrm{Gal}$ that is negligible with respect to the GRAIL noise level. 
The inversion was carried out in the same way as discussed for the simulated scenario (Section 3.2). However, we evaluated the inversion estimates of $\mathcal{T}^{0}$ and $\mathcal{T}^{1}$ with those from a topographically implied global GFM. Such a model can be taken as a proxy, because the observed lunar gravitational field and the one inferred by the lunar topography (and crust) are highly correlated beyond spherical harmonic degree 150 (e.g., Konopliv et al., 2014; Lemoine et al., 2014).

The topographically implied global GFM was determined by the forward modelling approach (Šprlák et al., 2018) with a constant density of $2550 \mathrm{~kg} \mathrm{~m}^{-3}$ for the lunar crust. More complex $3 \mathrm{D}$ crustal density distributions, such as radially and laterally variable, were tested and we did not find any contradictory conclusions to those made from a constant density. We also synthesised $\mathcal{T}^{0}$ and $\mathcal{T}^{1}$ from GRAIL-derived global GFMs with the highest available resolutions. Namely, we used GL1500E (available up to degree 1500, but exploited up to degree 1400) and GRGM1200A (available up to degree 1200) (Lemoine et al., 2014), and compared them with their topographically inferred counterparts.

The maps of $\mathcal{T}^{0}$ and their differences with respect to those from the inversion, GL1500E, and GRGM1200A are depicted in Fig. 6. The differences for the inversion are less than 15\% of the signal within the computation area, i.e., inside the red dashed box in Fig. 6b. The estimates outside the computation area are more erroneous and eventually unrealistic, because the mathematical model is not fulfilled there as the spherical caps extend beyond the data areas.

Figures $6 \mathrm{c}$ and $6 \mathrm{~d}$ show the performance of GL1500E and GRGM1200A models up to degrees 1400 and 1200. The values of $\mathcal{T}^{0}$ from GL1500E possess high-frequency noise and the differences extend beyond those for the inversion. High-frequency noise is also present in GRGM1200A, but this model appears to perform "better" than GL1500E. This could be explained by the application of the decreasing power law in GRGM1200A, as compared to the constant power law constraint used in GL1500E. The noise levels for GL1500E and GRGM1200A also differ due to the maximum harmonic degree of the respective syntheses.

The high-frequency noise of GL1500E and GRGM1200A is attenuated by truncating the spherical harmonic syntheses at degree 700, see Figs. 6e and 6 f. The differences for both models are almost identical at this (spectral and spatial) resolution, which demonstrates their comparable performance. The magnitudes of the differences also show that GL1500E and GRGM1200A are closer to the topographically inferred global GFM than the estimates from our regional inversion.

Figure 7 illustrates maps of $\mathcal{T}^{1}$ (from the topographically inferred global GFM) and its respective differences with the inversion, GL1500E and GRGM1200A. We observe similar performance among the three global GFMs and the inversion as in Fig. 6. However, the relative errors (i.e., the ratio of the magnitudes of the differences to those of the signal) are higher as compared to those for $\mathcal{T}^{0}$. For example, the differences for the inversion are about $30 \%$ of the signal amplitudes within the computation area (see Fig. 7b), while a similar comparison showed less than $15 \%$ for $\mathcal{T}^{0}$, see Fig. $6 \mathrm{~b}$. This behaviour can be explained by the stronger sensitivity of $\mathcal{T}^{1}$ to higher-frequencies of the gravitational field that also amplifies errors or noise.

We also investigated the equiangular grids from the inversion and the three global GFMs in the spectral domain. That is, we calculated the local degree variances by 2D Fourier transform and azimuthal averaging (Flury, 2006) from the six grids in the computation area. The local signal spectra for $\mathcal{T}^{0}$ are depicted in Fig. 8 and are almost identical up to degree $\sim 360$. Above this degree, the signal of the inversion grid (red) slightly decreases, but stays approximately parallel to the topographically inferred one (black) even for high degrees. GL1500E (solid green) and GRGM1200A (solid blue) spectra of the high-resolution grids depart from the topographically inferred one above degree $\sim 450$ and the distinctions are significant beyond degree 600 . The spectra for the lower resolution grids from GL1500E (dashed green) and GRGM1200A (dashed blue) follow its topographical equivalent to degree $\sim 600$. The lack of the power for the lower resolution grids is evident beyond this degree and magnitudes decrease below the one for the 
a)

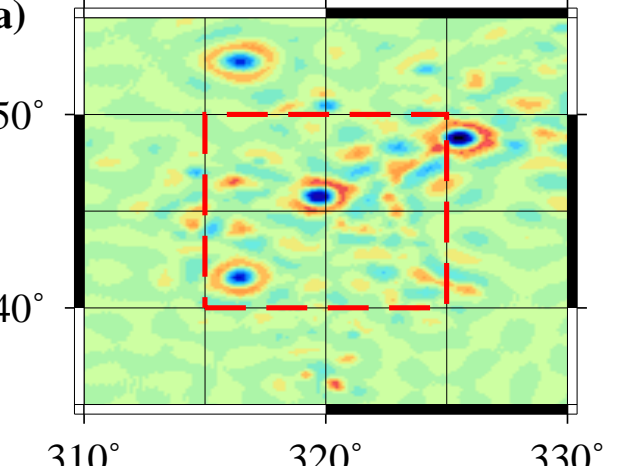

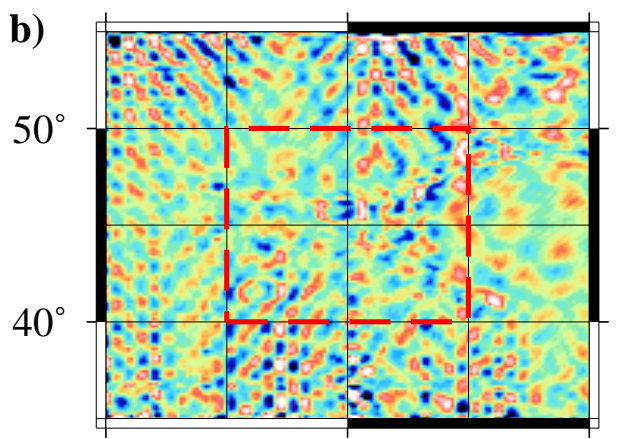
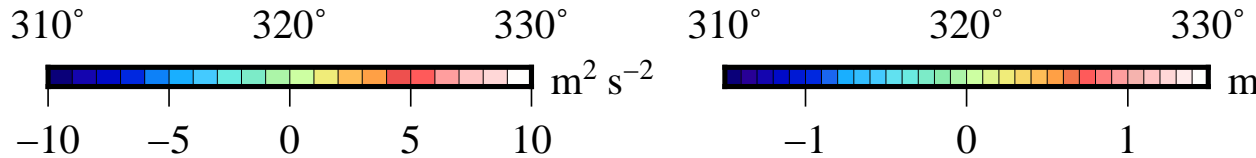

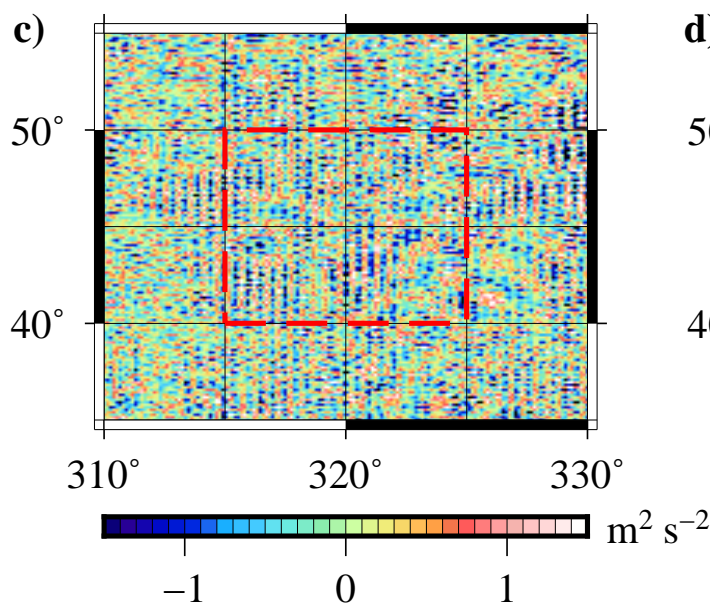

$-1$

e)

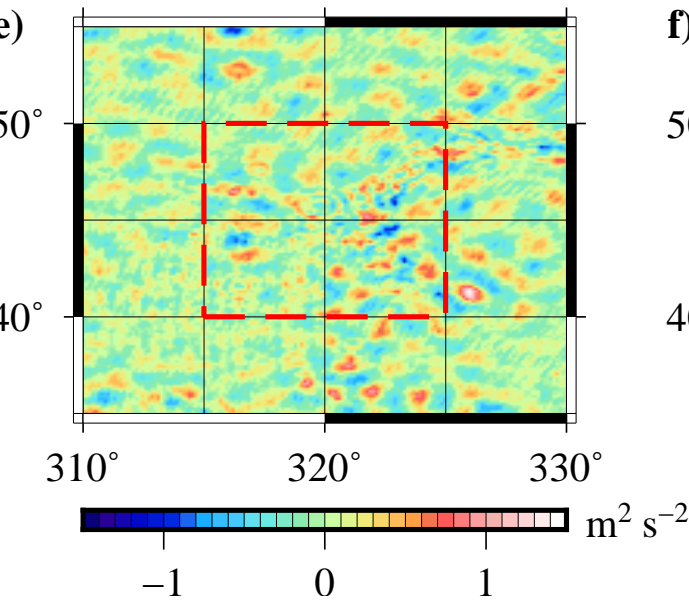

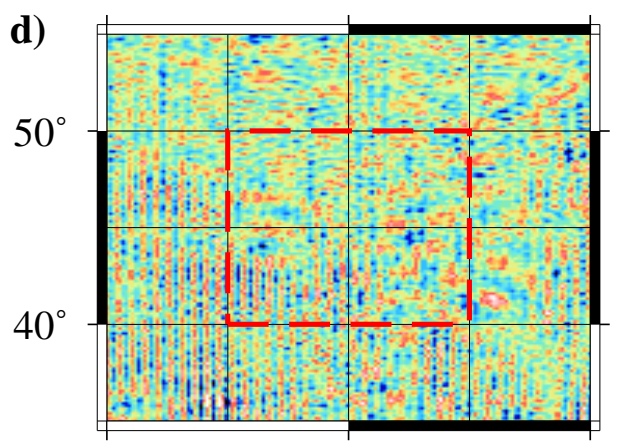
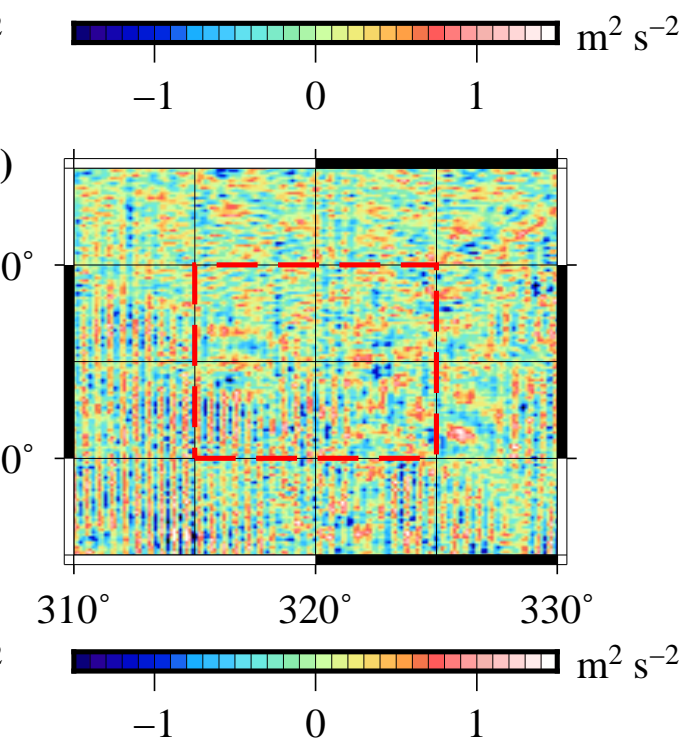

f)
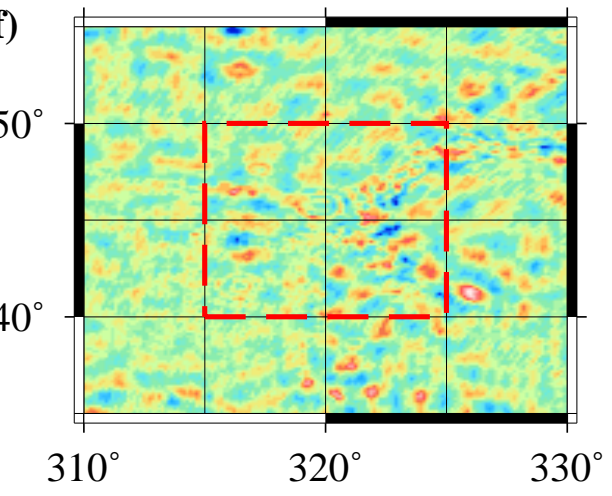

Figure 6: Maps of: a) the topographically implied $\mathcal{T}^{0}$ (between degrees 200-1400) and its differences with respect to b) inversion, c) GL1500E (between degrees 200-1400), d) GRGM1200A (between degrees 200-1200), e) GL1500E (between degrees 200-700), and f) GRGM1200A (between degrees 200-700). The red dashed line bounds the computation area.

inversion grid at $\sim 720$.

Overall, the results for our localised inversion method show some reasonable performance. The mutual agreement between two GRAIL global GFMs up to degree 500 are rather expected because they exploit the same mathematical apparatus of spherical harmonic basis functions 

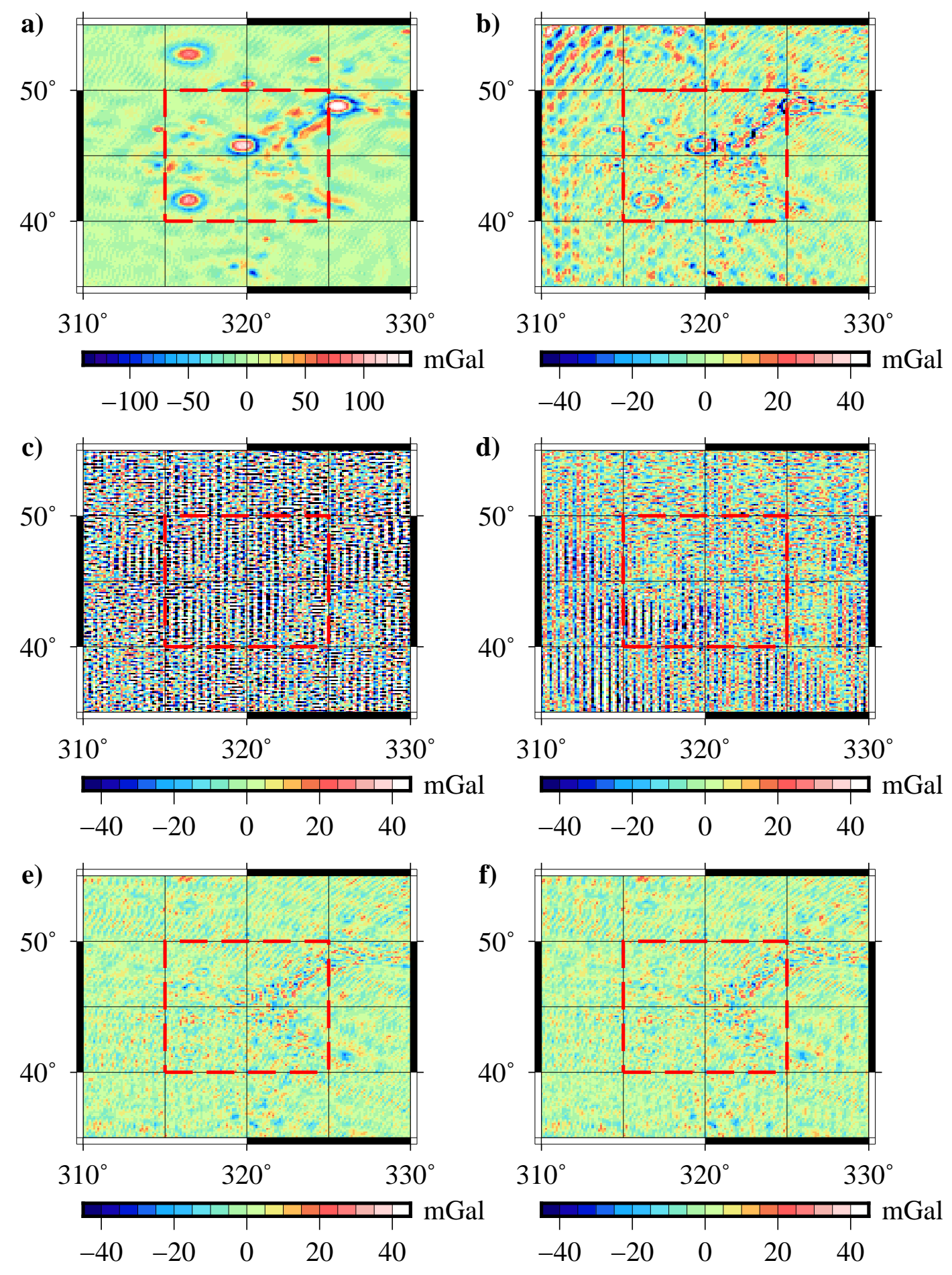

Figure 7: Same as in Fig. 6, but for $\mathcal{T}^{1}$.

for representing gravitational fields and the identical approaches of numerical force integration for analysing the inter-satellite ranging measurements. On the other hand, our approach is truly independent from the existing GRAIL solutions in terms of the spatial (not spectral) parametrisation of gravitational fields and the use of LoS gravitational acceleration as observable. Finally, there are some other inconsistencies arising from the comparison with the topographyinferred global GFM. For example, point values were synthesised from the topography-inferred 


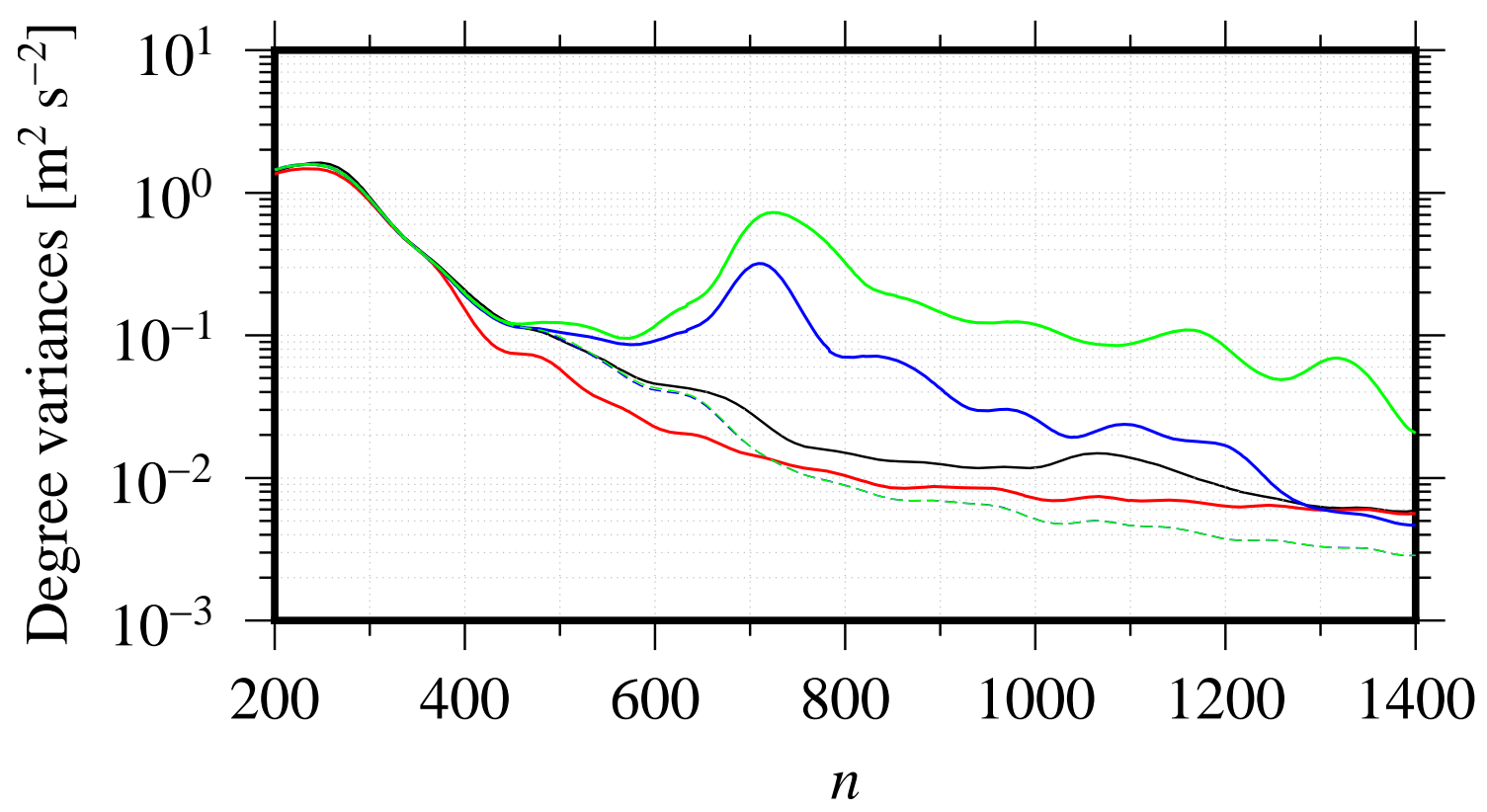

Figure 8: Local degree variances for the grids of $\mathcal{T}^{0}$ calculated by the topographically inferred GGFM between degrees 200-1400 (black), inversion (red), GL1500E between degrees 200-1400 (solid green), GRGM1200A between degrees 200-1200 (solid blue), GL1500E between degrees 200-700 (dashed green), and GRGM1200A between degrees 200-700 (dashed blue).

global GFM, while integral inversion provided averages over individual cells. However, rigorous evaluation of either point or mean values by integral inversion is a more complex subject (Huang, 2002) that is out of the scopes of this study.

\section{Summary and Conclusions}

An independent approach for regional recovery of the lunar gravitational field from GRAILsensed inter-satellite-tracking observables was presented in this article. We derived integral transformations relating the line-of-sight ( $\mathrm{LoS})$ disturbing gravitational acceleration to an arbitraryorder radial derivative of the disturbing gravitational potential (Eq. (23)). We expressed the truncation error (the neglected effect of the far zones beyond a spherical cap centred on each computation point) of the LoS disturbing gravitational acceleration by a spherical harmonic expansion of Eq. (37). For practical calculations, we formulated remove-compute-restore estimators to transform an arbitrary-order radial derivative of the disturbing gravitational potential onto the LoS disturbing gravitational acceleration (Eq. (38)). Such mathematical apparatus is not only restricted to the lunar GRAIL mission, but also can be exploited for GRACE and GRACE-FO.

Numerical experiments were conducted to investigate the performance of the integral estimators when solving upward and downward continuation problems for the high-resolution lunar gravitational field. The presented experiments were restricted to the disturbing gravitational potential and its first radial derivative for the sake of brevity, though they may hold for other relations in the Meissl scheme (Meissl, 1971; Rummel and van Gelderen, 1995). We tested the accuracy of the integral estimators for the maximum spherical harmonic degree of the low-frequency part of the lunar gravitational field $N=199$. Optimal integration radii and discretisation intervals were found empirically, for which the GRAIL noise level dominated the accuracy of the integral estimators. Finally, we tested the performance of the integral inversion with synthetic and realistic GRAIL observations. The realistic regional gravitational fields 
and those of GL1500E and GRGM1200A were validated with the topography-implied global gravitational field model, acknowledging the underlying assumptions of a constant crustal density and no isostatic compensation. We demonstrated that the new regional solutions are less contaminated by high-frequency noise than the existing global gravitational field solutions.

Acknowledgement: This research was supported financially by the Australian Government through the Australian Research Council's Discovery Projects funding scheme (project DP160104095). Thoughtful and constructive comments of the two anonymous reviewers are gratefully acknowledged. Thanks are also extended to the editor-in-chief Dr. Pascal Willis and the responsible editor DR. Anil Bhardwaj for handling our manuscript.

\section{Appendix A. Spatial forms of isotropic kernels}

We summarise the spatial (closed, analytical) forms of the isotropic kernels in this Appendix. We restrict to the values of $k=0,1,2,3$, and use the substitution $g=g(t, u)=\sqrt{1-2 t u+t^{2}}$. The spatial forms can be derived by decomposing the degree-dependent terms of the corresponding spectral relations into partial fractions. Each partial fraction is an infinite series, the closed form of which is found by summation rules given in, e.g., (Pick et al., 1973; Martinec, 2003; Šprlák and Novák, 2014).

The spatial forms of the isotropic kernels for the radial BVP represented by the spectral relation of Eq. (13) read (Heiskanen and Moritz, 1967; Hotine, 1969; Martinec, 2003; Šprlák and Novák, 2016):

$$
\begin{aligned}
& \mathcal{H}_{0}(t, u)=\frac{t\left(1-t^{2}\right)}{g^{3}}, \\
& \mathcal{H}_{1}(t, u)=\frac{2 t}{g}-\ln \left(\frac{g+t-u}{1-u}\right), \\
& \mathcal{H}_{2}(t, u)=\frac{3}{t}(g-1)+\left(\frac{3 u}{t}-1\right) \ln \left(\frac{g+t-u}{1-u}\right), \\
& \mathcal{H}_{3}(t, u)=\frac{1}{4 t}\left\{7 g-12-\frac{15 u(g-1)}{t}-\frac{1}{t}\left[2 t(t-6 u)-5\left(1-3 u^{2}\right)\right] \ln \left(\frac{1+u}{g-t+u}\right)\right\} .
\end{aligned}
$$

The Abel-Poisson kernel is defined by Eq. (A.1) and the extended Hotine kernel (Hotine, 1969) is given by Eq. (A.2). Equation (A.3) is the isotropic kernel of the vertical-vertical gradiometric BVP (Martinec, 2003). The isotropic kernel of the vertical-vertical-vertical gravitational curvature BVP is given by Eq. (A.4).

The isotropic kernels $\mathcal{H}_{k}^{t}, k=0,1,2,3$, are required for the integral transformations of the radial disturbing gravitational potential derivatives onto the LoS gravitational acceleration, see Eq. (24). Their closed forms read as:

$$
\begin{aligned}
& \mathcal{H}_{0}^{t}(t, u)=-\frac{t^{2}}{g^{3}}\left[1+3 t(u-2 t)+\frac{6 t^{2}(t-u)^{2}}{g^{2}}\right], \\
& \mathcal{H}_{1}^{t}(t, u)=-\frac{t^{2}\left(1-t^{2}\right)}{g^{3}},
\end{aligned}
$$


$\mathcal{H}_{2}^{t}(t, u)=3(g-1)-\frac{2 t^{2}}{g}+3 y \ln \left(\frac{1+u}{g-t+u}\right)$

$\mathcal{H}_{3}^{t}(t, u)=\frac{1}{2 t}\left[t(g-6)+15 u(1-g)+[5+3 u(2 t-5 u)] \ln \left(\frac{1+u}{g-t+u}\right)\right]$.

The isotropic kernels in Eqs. (A.5)-(A.8) are always bounded for $u \in[-1,1]$ and $t<1$. In particular, we can find the following limits when the computation point and the integration point coincide (i.e., $u=1$ ):

$\mathcal{H}_{0}^{t}(t, 1)=-\frac{t^{2}(1+3 t)}{(1-t)^{3}}, \quad \mathcal{H}_{1}^{t}(t, 1)=-\frac{t^{2}(1+t)}{(1-t)^{2}}, \quad \mathcal{H}_{2}^{t}(t, 1)=\frac{t(t-3)}{1-t}+3 \ln \left(\frac{1}{1-t}\right)$,

$\mathcal{H}_{3}^{t}(t, 1)=\frac{1}{2}(10-t)+\frac{1}{t}(3 t-5) \ln \left(\frac{1}{1-t}\right)$.

For $u=-1$ (i.e., at the anti-computational point), we obtain bounded limiting values:

$\mathcal{H}_{0}^{t}(t,-1)=-\frac{t^{2}(1-3 t)}{(1+t)^{3}}, \quad \mathcal{H}_{1}^{t}(t,-1)=-\frac{t^{2}(1-t)}{(1+t)^{2}}, \quad \mathcal{H}_{2}^{t}(t,-1)=\frac{t(t+3)}{1+t}+3 \ln \left(\frac{1}{1+t}\right)$,

$\mathcal{H}_{3}^{t}(t,-1)=\frac{1}{2}(10+t)+\frac{1}{t}(3 t+5) \ln \left(\frac{1}{1+t}\right)$.

Also, the isotropic kernels in Eq. (25) are necessary when transforming the radial disturbing gravitational potential derivatives onto the LoS gravitational acceleration. Their analytical expressions are of the form:

$$
\begin{aligned}
& \mathcal{H}_{0}^{u}(t, u)=\frac{3 t^{3} \sqrt{1-u^{2}}}{g^{3}}\left[1+\frac{2 t(u-t)}{g^{2}}\right], \\
& \mathcal{H}_{1}^{u}(t, u)=t \sqrt{1-u^{2}}\left\{\frac{2 t^{2}}{g^{3}}+\frac{1}{g+t-u}\left[1+\frac{t}{g}-\frac{1+u}{g-t+u}\right]\right\}, \\
& \mathcal{H}_{2}^{u}(t, u)=-\sqrt{1-u^{2}}\left\{\frac{3}{g}\left[t+\frac{u(1+t)}{1+u}-\frac{u}{g-t+u}\right]-\frac{t}{g+t-u}\left[1+\frac{t}{g}-\frac{1+u}{g-t+u}\right]\right. \\
& \left.-3 \ln \left(\frac{1+u}{g-t+u}\right)\right\}, \\
& \mathcal{H}_{3}^{u}(t, u)=\frac{\sqrt{1-u^{2}}}{2 t}\left\{5(3-2 g+u)-\frac{1}{g}\left[t^{2}-5 u(t+u)+6 t u\left(\frac{1+t}{1+u}-\frac{1}{g-t+u}\right)\right]\right. \\
& \left.+\frac{1}{g+t-u}\left[t^{2}+\frac{5 u^{3}+t^{3}}{g}-\frac{(1+u)\left(5+t^{2}\right)}{g-t+u}\right]-3(5 u-2 t) \ln \left(\frac{1+u}{g-t+u}\right)\right\} .
\end{aligned}
$$

Equations (A.11)-(A.14) represent bounded functions for $u \in[-1,1]$ and $t<1$, with the limiting values at $u= \pm 1$ :

$\mathcal{H}_{k}^{u}(t, 1)=\mathcal{H}_{k}^{u}(t,-1)=0, \quad k=0,1,2,3$. 


\section{References}

Akim EL (1966) Determination of the gravitational field of the Moon from the motion of the artificial lunar satellite Luna-10. Doklady Akademii Nauk SSSR 170:799-802.

Ardalan AA, Karimi R (2014) Effect of topographic bias on geoid and reference ellipsoid of Venus, Mars, and the Moon. Celestial Mechanics and Dynamical Astronomy 118:75-88. doi: https://doi.org/10.1007/s10569-013-9523-6.

Barriot JP, Balmino G (1992) Estimation of local planetary gravity fields using line of sight gravity data and an integral operator. Icarus 99:202-224. doi: https://doi.org/10.1016/00191035(92)90183-8.

Bentel K, Schmidt M, Gerlach C (2013) Different radial basis functions and their applicability for regional gravity field representation on the sphere. GEM - International Journal on Geomathematics 4:67-96. doi: https://doi.org/10.1007/s13137-012-0046-1.

Bouman J (1998) Quality of regularization methods. DEOS Report No. 98.2, Delft University Press, Delft, The Netherlands, 104 pp.

Cai L, Zhou Z, Hsu H, Gao F, Zhu Z, Luo J (2013) Analytical error analysis for satellite gravity field determination based on two-dimensional Fourier method. Journal of Geodesy 87:417-426. doi: https://doi.org/10.1007/s00190-013-0615-6.

Casotto S, Fantino E (2009) Gravitational gradients by tensor analysis with application to spherical coordinates. Journal of Geodesy 83:621-634. doi: https://doi.org/10.1007/s00190008-0276-z.

Chauvenet W (1875) A treatise on plane and spherical trigonometry, Ninth Edition. JB Lippincott \& Co., Philadelphia, U.S.A., 270 pp.

Eshagh M (2011) The effect of spatial truncation error on integral inversion of satellite gravity gradiometry data. Advances in Space Research 47:1238-1247. doi: https://doi.org/10.1016/j.asr.2010.11.035.

Eshagh M, Šprlák M (2016) On the integral inversion of satellite-to-satellite velocity differences for local gravity field recovery: a theoretical study. Celestial Mechanics and Dynamical Astronomy 124:127-144. doi: https://doi.org/10.1007/s10569-015-9654-z.

Evans JD, Featherstone WE (2000) Improved convergence rates for the truncation error in gravimetric geoid determination. Journal of Geodesy 74:239-248. doi: https://doi.org/10.1007/s001900050282.

Floberghagen R (2002) Lunar gravimetry. Revealing the far-side. Kluwer Academic Publishers, Dordrecht, The Netherlands, 304 pp., doi: https://doi.org/10.1007/978-90-481-9552-7.

Floberghagen R, Bouman J, Koop R, Visser PNAM (1999) On the information contents and regularisation of the lunar gravity field solutions. Advances in Space Research 23:1801-1807. doi: https://doi.org/10.1016/S0273-1177(99)00534-7.

Flury J (2006) Short-wavelength spectral properties of the gravity field from a range of regional data sets. Journal of Geodesy 79:624-640. doi: https://doi.org/10.1007/s00190-005-0011-y.

Forsberg R, Olesen AV (2010) Airborne gravity field determination. In: Xu G (Ed.) Sciences of Geodesy - I: Advances and Future Directions, Springer-Verlag, Berlin, Germany, pp. 83-104. 
Freeden W, Michel V, Nutz H (2002) Satellite-to-satellite tracking and satellite gravity gradiometry (Advanced techniques for high-resolution geopotential field determination). Journal of Engineering Mathematics 43:19-56. doi: https://doi.org/10.1023/A:1016577524288.

Freeden W, Nashed MZ (2018) Operator-theoretic and regularization approaches to ill-posed problems. International Journal of Geomathematics 9:1-115. doi: https://doi.org/10.1007/s13137-017-0100-0.

Garcia RV (2002) Local geoid determination from GRACE mission. Report No. 460, Department of Civil and Environmental Engineering and Geodetic Science, The Ohio State University, Columbus, Ohio, U.S.A., 106 pp.

Grafarend EW (2006) Linear and nonlinear models: fixed effects, random effects, and mixed models. Walter de Gruyter, Berlin, Germany, 752 pp.

Grafarend EW, Finn G, Ardalan AA (2006) Ellipsoidal vertical deflections and ellipsoidal gravity disturbance: case studies. Studia Geophysica et Geodaetica 50:1-57. doi: https://doi.org/10.1007/s11200-006-0001-4.

Gruber C, Moon Y, Flechtner F, Dahle C, Novák P, König R, Neumayer H (2014) Submonthly GRACE solutions from localizing integral equations and Kalman filtering. In: Rizos C, Willis $\mathrm{P}$ (eds) Earth on the Edge: Science for a Sustainable Planet. International Association of Geodesy Symposia, Vol. 139. Springer, Berlin, Heidelberg, Germany, pp. 383-389. doi: https://doi.org/10.1007/978-3-642-37222-3_51.

Goossens S, Visser PNAM, Ambrosius BAC (2005a) A method to determine regional lunar gravity fields from earth-based satellite tracking data. Planetary and Space Science 53:1331-1340. doi: https://doi.org/10.1016/j.pss.2005.06.009.

Goossens S, Visser PNAM, Heki K, Ambrosius BAC (2005b) Local gravity from Lunar Prospector tracking data: Results for Mare Serenitatis. Earth, Planets and Space 57:1127-1132. doi: https://doi.org/10.1186/BF03351893.

Goossens S, Ishihara Y, Matsumoto K, Sasaki S (2012) Local lunar gravity field analysis over the South Pole-Aitken basin from SELENE farside tracking data. Journal of Geophysical Research 117:E02005. doi: https://doi.org/10.1029/2011JE003831.

Goossens S, Sabaka TJ, Nicholas JB, Lemoine FG, Rowlands DD, Mazarico E, Neumann GA, Smith DE, Zuber MT (2014) High-resolution local gravity model of the south pole of the Moon from GRAIL extended mission data. Geophysical Research Letters 41:3367-3374. doi: https://doi.org/10.1002/2014GL060178.

Hagiwara Y (1972) Truncation error formulas for the geoidal height and the deflection of the vertical. Bulletin Géodésique 106:453-466. doi: https://doi.org/10.1007/BF02522052.

Hajela DP (1979) Tests for the recovery of $5^{\circ}$ mean gravity anomalies in local areas from ATS 6/GEOS 3 satellite to satellite range-rate observations. Journal of Geophysical Research 84:6884-6890. doi: https://doi.org/10.1029/JB084iB12p06884.

Han S-C (2008) Improved regional gravity fields on the Moon from Lunar Prospector tracking data by means of localized spherical harmonic functions. Journal of Geophysical Research 113: E11012. doi: https://doi.org/10.1029/2008JE003166.

Han S-C (2013) Determination and localized analysis of intersatellite line of sight gravity difference: Results from the GRAIL primary mission. Journal of Geophysical Research - Planets 118:2323-2337. doi: https://doi.org/10.1002/2013JE004402. 
Han S-C, Mazarico E, Rowlands D, Lemoine F, Goossens S (2011) New analysis of Lunar Prospector radio tracking data brings the nearside gravity field of the Moon with an unprecedented resolution. Icarus 215: 455-459. doi: https://doi.org/10.1016/j.icarus.2011.07.020.

Han S-C, Mazarico E, Lemoine F (2009) Improved nearside gravity field of the Moon by localizing the power law constraint. Geophysical Research Letters 36:L11203. doi: https://doi.org/10.1029/2009GL038556.

Hansen PC (1998) Rank-deficient and discrete ill-posed problems. SIAM Monographs on Mathematical Modeling and Computation. Philadelphia, U.S.A., 247 pp.

Hansen PC (2007) Regularization Tools version 4.0 for Matlab 7.3. Numerical Algorithms 46:189194. doi: https://doi.org/10.1007/s11075-007-9136-9.

Heiskanen WA, Moritz H (1967) Physical geodesy. Freeman and Co., San Francisco, U.S.A., 364 pp.

Hobson EW (1965) The theory of spherical and ellipsoidal harmonics. Chelsea Publishing Company, New York, U.S.A., 500 pp.

Hotine M (1969) Mathematical geodesy. Environmental Science Services Administration, Monograph No. 2, US Department of Commerce, Washington DC, U.S.A., 416 pp.

Huang J (2002) Computational methods for the discrete downward continuation of the Earth gravity and effects of lateral topographical mass density variation on gravity and the geoid. Technical Report No. 16, Department of Geodesy and Geomatics Engineering, University of New Bruinswick, Fredericton, Canada, 141 pp.

Jekeli C (1979) Global accuracy estimates of point and mean undulation differences obtained from gravity disturbances, gravity anomalies and potential coefficients. Report No. 288, Department of Geodetic Science and Surveying, The Ohio State University, Columbus, U.S.A., $41 \mathrm{pp}$.

Jekeli C (1981) Modifying Stokes' function to reduce the error of geoid undulation computations. Journal of Geophysical Research 86:6985-6990. doi: https://doi.org/10.1029/JB086iB08p06985.

Kaula WM (1966) Theory of satellite geodesy: Applications of satellite to Geodesy. Dover Publications, Inc., New York, U.S.A., 124 pp.

Keller W, Sharifi MA (2005) Satellite gradiometry using a satellite pair. Journal of Geodesy 78:544-557. doi: https://doi.org/10.1007/s00190-004-0426-x.

Kellogg OD (1929) Foundations of potential theory. Verlag von Julius Springer, Berlin, Germany, 384 pp.

Kingdon R, Vaníček P (2011) Poisson downward continuation solution by the Jacobi method. Journal of Geodetic Science 1:74-81. doi: https://doi.org/10.2478/v10156-010-0009-0.

Klinger B, Baur O, Mayer-Gürr T (2014) GRAIL gravity field recovery based on the shortarc integral equation technique: Simulation studies and first real data results. Planetary and Space Science 91:83-90. doi: https://doi.org/10.1016/j.pss.2013.12.001.

Koch KR, Kusche J (2002) Regularization of geopotential determination from satellite data by variance components. Journal of Geodesy 76:259-268. doi: https://doi.org/10.1007/s00190002-0245-x. 
Konopliv AS, Binder AB, Hood LL, Kucinskas AB, Sjogren WL, Williams JG (1998) Improved gravity field of the moon from Lunar Prospector. Science 281:1476-1480. doi: https://doi.org/10.1126/science.281.5382.1476.

Konopliv AS, Asmar SW, Carranza E, Sjogren WL, Yuan D-N (2001) Recent gravity models as a result of the Lunar Prospector mission. Icarus 150:1-18. doi: https://doi.org/10.1006/icar.2000.6573.

Konopliv AS, Park RS, Yuan D-N, Asmar SW, Watkins MM, Williams JG, Fahnestock E, Kruizinga G, Paik M, Strekalov D, Harvey N, Smith DE, Zuber MT (2013) The JPL lunar gravity field to spherical harmonic degree 660 from the GRAIL Primary Mission. Journal of Geophysical Research - Planets 118:1415-1434. doi: https://doi.org/10.1002/jgre.20097.

Konopliv AS, Park RS, Yuan D-N, Asmar SW, Watkins MM, Williams JG, Fahnestock E, Kruizinga G, Paik M, Strekalov D, Harvey N, Smith DE, Zuber MT (2014) High-resolution lunar gravity fields from the GRAIL Primary and Extended Missions. Geophysical Research Letters 41:1452-1458. doi: https://doi.org/10.1002/2013GL059066.

Lemoine FG, Goossens S, Sabaka TJ, Nicholas JB, Mazarico E, Rowlands DD, Loomis BD, Chinn DS, Caprette DS, Neumann GA, Smith DE, Zuber MT (2013) High-degree gravity models from GRAIL primary mission data. Journal of Geophysical Research - Planets 118:1676-1699. doi: https://doi.org/10.1002/jgre.20118.

Lemoine FG, Goossens S, Sabaka TJ, Nicholas JB, Mazarico E, Rowlands DD, Loomis BD, Chinn DS, Neumann GA, Smith DE, Zuber MT (2014) GRGM900C: A degree 900 lunar gravity model from GRAIL primary and extended mission data. Geophysical Research Letters 41:3382-3389. doi: https://doi.org/10.1002/2014GL060027.

Martinec Z (2003) Green's function solution to spherical gradiometric boundary-value problems. Journal of Geodesy 77:41-49. doi: https://doi.org/10.1007/s00190-002-0288-z.

McKenzie D, Nimmo F (1997) Elastic thickness estimates for Venus from line of sight accelerations. Icarus 130: 198-216. doi: https://doi.org/10.1006/icar.1997.5770.

Meissl P (1971) A study of covariance functions related to the Earth's disturbing potential. Report No. 151, Department of Geodetic Science, The Ohio State University, Columbus, Ohio, U.S.A, 88 pp.

Miller K (1970) Least squares methods for ill-posed problems with a prescribed bound. SIAM Journal on Mathematical Analysis 1:52-74. doi: https://doi.org/10.1137/0501006.

Molodensky MS, Eremeev VF, Yurkina MI (1962) Methods for study of the external gravitational field and figure of the Earth. The Israel Program for Scientific Translations, Department of Commerce, Washington DC, U.S.A., 248 pp.

Muller PM, Sjogren WL (1968) Mascons: lunar mass concentrations. Science 61:680-684. doi: https://doi.org/10.1126/science.161.3842.680.

Namiki N, Iwata T, Matsumoto K, Hanada H, Noda H, Goossens S, Ogawa M, Kawano N, Asari K, Tsuruta S, Ishihara Y, Liu Q, Kikuchi F, Ishikawa T, Sasaki S, Aoshima C, Kurosawa K, Sugita S, Takano T (2009) Farside gravity field of the Moon from four-way Doppler measurements of SELENE (Kaguya). Science 323:900-905. doi: https://doi.org/10.1126/science.1168029. 
Novák P (2007) Integral inversion of SST data of type GRACE. Studia Geophysica et Geodaetica 51:351-367. doi: https://doi.org/10.1007/s11200-007-0020-9.

Novák P, Vaníček P, Véronneau M, Holmes S, Featherstone W (2001) On the accuracy of modified Stokes's integration in high-frequency gravimetric geoid determination. Journal of Geodesy 74:644-654. doi: https://doi.org/10.1007/s001900000126.

Paul M (1973) A method of evaluation the truncation error coefficients for geoidal heights. Bulletin Géodésique 110:413-425. doi: https://doi.org/10.1007/BF02521951.

Pavlis NK (1991) Estimation of geopotential differences over intercontinental locations using satellite and terrestrial measurements. Report No. 409, Department of Geodetic Science and Surveying, The Ohio State University, Columbus, U.S.A., 155 pp.

Phillips DL (1962) A technique for the numerical solution of certain integral equations of the first kind. Journal of the Association for Computational Machinery 9: 84-96. doi: https://doi.org/10.1145/321105.321114.

Pick M, Pícha J, Vyskočil V (1973) Theory of the Earth's gravity field. Elsevier, Amsterdam, The Netherlands, 538 pp.

Rapp RH, Rummel R (1975) Methods for the computation of detailed geoids and their accuracy. Report No. 233, Department of Geodetic Science, Ohio State University, Columbus, U.S.A., $36 \mathrm{pp}$.

Reigber C, Lühr H, Schwintzer P (2002) CHAMP mission status. Advances in Space Research 30:129-134. doi: https://doi.org/10.1016/S0273-1177(02)00276-4.

Rummel R (1980) Geoid heights, geoid height differences, and mean gravity anomalies from 'low-low' satellite-to-satellite tracking - an error analysis. Report No. 306, Department of Geodetic Science, Ohio State University, Columbus, U.S.A., 44 pp.

Rummel R, van Gelderen M (1995) Meissl scheme - spectral characteristics of physical geodesy. manuscripta geodaetica 20:379-385.

Sansò F, Sideris MG (2013) Geoid determination. Lecture Notes in Earth System Sciences, Vol. 110, Springer-Verlag, Berlin Heidelberg, Germany, 734 pp.

Schmidt M, Fengler M, Mayer-Gürr T, Eicker A, Kusche J, Sánchez L, Han S-C (2007) Regional gravity modeling in terms of spherical base functions. Journal of Geodesy 81:17-38. doi: https://doi.org/10.1007/s00190-006-0101-5.

Shen Y, Xu P, Li B (2012) Bias-corrected regularized solution to inverse ill-posed models. Journal of Geodesy 86: 597-608. doi: https://doi.org/10.1007/s00190-012-0542-y.

Simmonds JG (1994) A brief on tensor analysis, Second edition. Undergraduate Texts in Mathematics, Springer-Verlag, New York, U.S.A., 112 pp.

Sjöberg LE (2003) A general model of modifying Stokes' formula and its least-squares solution. Journal of Geodesy 77:459-464. doi: https://doi.org/10.1007/s00190-003-0346-1.

Sjöberg LE, Featherstone WE (2004) Two-step procedures for hybrid geoid modelling. Journal of Geodesy 78:66-75. https://doi.org/10.1007/s00190-003-0367-9.

Sjogren WL, Wimberly RN, Wollenhaupt WR (1974) Lunar gravity via the Apollo 15 and 16 subsatellites. Moon 9:115-128. doi: https://doi.org/10.1007/BF00565398. 
Šprlák M (2010) Generalized geoidal estimators for deterministic modifications of spherical Stokes's function. Contributions to Geophysics and Geodesy 40:45-64. doi: https://doi.org/10.2478/v10126-010-0003-7.

Šprlák M, Han S-C, Featherstone W (2018) Forward modelling of global gravity fields with 3D density structures and an application to the high-resolution $(\sim 2 \mathrm{~km})$ gravity fields of the Moon. Journal of Geodesy 92:847-862. doi: https://doi.org/10.1007/s00190-017-1098-7.

Šprlák M, Novák P (2014) Integral transformations of gradiometric data onto a GRACE type of observable. Journal of Geodesy 88:377-390. doi: https://doi.org/10.1007/s00190-013-0689-1.

Šprlák M, Novák P (2016) Spherical gravitational curvature boundary-value problem. Journal of Geodesy 90:727-739. doi: https://doi.org/10.1007/s00190-016-0905-x.

Sugano T, Heki K (2004) High resolution gravity anomaly map from the Lunar Prospector line-of-sight acceleration data. Earth, Planets and Space 56:81-86. doi: https://doi.org/10.1186/BF03352492.

Sünkel H (1981) Feasibility studies for the prediction of the gravity disturbance vector in high altitudes. Report No. 311, Department of Geodetic Science and Surveying, The Ohio State University, Columbus, U.S.A., 53 pp.

Tapley BD, Bettadpur S, Watkins M, Reigber C (2004) The gravity recovery and climate experiment: Mission overview and early results. Geophysical Research Letters 31:L09607. doi: https://doi.org/10.1029/2004GL019920.

Tenzer R, Novák P (2008) Conditionality of inverse solutions to discretised integral equations in geoid modelling from local gravity data. Studia Geophysica et Geodaetica 52:53-70. doi: https://doi.org/10.1007/s11200-008-0005-3.

Tenzer R, Foroughi I, Sj'oberg LE, Bagherbandi M, Hirt C, Pitoňák (2018) Surveys in Geophysics 39(3): 313-335. doi: https://doi.org/10.1007/s10712-017-9457-8.

Thalhammer M (1994) The geographical truncation error in satellite gravity gradiometer measurements. manuscripta geodaetica 19:45-54.

Tikhonov AN (1963a) Solution of incorrectly formulated problems and the regularization method. Soviet Mathematics - Doklady 4:1035-1038.

Tikhonov AN (1963b) Regularization of incorrectly posed problems. Soviet Mathematics - Doklady 4:1624-1627.

Timmen L (2010) Absolute and relative gravimetry. In: Xu G (Ed.) Sciences of Geodesy - I: Advances and Future Directions, Springer-Verlag, Berlin, Germany, pp. 1-48.

Torge W (1989) Gravimetry. De Gruyter, Berlin, New York, 465 pp.

Torge W, Müller J (2012) Geodesy, 4th Edition. De Gruyter Inc., Berlin, Boston, 433 pp.

Vaníček P, Featherstone WE (1998) Performance of three types of Stokes's kernel in the combined solution for the geoid. Journal of Geodesy 72:684-697. doi: https://doi.org/10.1007/s001900050209.

Wirnsberger H, Krauss S, Mayer-Gürr T (2019) First independent Graz Lunar Gravity Model derived from GRAIL. Icarus 317:324-336. doi: https://doi.org/10.1016/j.icarus.2018.08.011. 
Xu P (1998) Truncated SVD methods for discrete linear ill-posed problems. Geophysical Journal International 135:505-514. doi: https://doi.org/10.1046/j.1365-246X.1998.00652.x.

$\mathrm{Xu}$ P (2009) Iterative generalized cross-validation for fusing heteroscedastic data of inverse ill-posed problems. Geophysical Journal International 179:182-200. doi: https://doi.org/10.1111/j.1365-246X.2009.04280.x.

Xu P, Shen YZ, Fukuda Y, Liu Y (2006) Variance components estimation in linear inverse illposed models. Journal of Geodesy 80:69-81. doi: https://doi.org/10.1007/s00190-006-0032-1.

Zuber MT, Smith DE, Lemoine FG, Neumann GA (1994) The shape and internal structure of the Moon from the Clementine mission. Science 266:1839-1843. doi: https://doi.org/10.1126/science.266.5192.1839.

Zuber MT, Smith DE, Lehman DH, Hoffman TL, Asmar SW, Watkins MM (2013) Gravity Recovery and Interior Laboratory (GRAIL): mapping the lunar interior from crust to core. Space Science Reviews 178:3-24. doi: https://doi.org/10.1007/s11214-012-9952-7. 